\title{
Normal Aging Affects the Short-Term Temporal Stability of Implicit, But Not Explicit, Motor Learning following Visuomotor Adaptation
}

\author{
DCGuneet Bindra, ${ }^{1}$ Rylee Brower, ${ }^{1}$ Ryan North, ${ }^{1}$ Weiwei Zhou, ${ }^{1, *}$ and ${ }^{-W i l s a a n ~ M . ~ J o i n e r ~}{ }^{1,2, *}$
}

https://doi.org/10.1523/ENEURO.0527-20.2021

${ }^{1}$ Department of Neurobiology, Physiology and Behavior, University of California, Davis, Davis, California 95616 and

${ }^{2}$ Department of Neurology, University of California, Davis, Davis, California 95616

\begin{abstract}
Normal aging is associated with a decline in memory and motor learning ability. However, the exact form of these impairments (e.g., the short-term temporal stability and affected learning mechanisms) is largely unknown. Here, we used a sensorimotor adaptation task to examine changes in the temporal stability of two forms of learning (explicit and implicit) because of normal aging. Healthy young subjects (age range, 19-28 years; 20 individuals) and older human subjects (age range, 63-85 years; 19 individuals) made reaching movements in response to altered visual feedback. On each trial, subjects turned a rotation dial to select an explicit aiming direction. Once selected, the display was removed and subjects moved the cursor from the start position to the target. After initial training with the rotational feedback perturbation, subjects completed a series of probe trials at different delay periods to systematically assess the short-term retention of learning. For both groups, the explicit aiming showed no significant decrease over $1.5 \mathrm{~min}$. However, this was not the case for implicit learning; the decay pattern was markedly different between groups. Older subjects showed a linear decrease of the implicit component of adaptation over time, while young subjects showed an exponential decay over the same period (time constant, $25.61 \mathrm{~s}$ ). Although older subjects adapted at a similar rate, these results suggest natural aging selectively impacts the short-term (seconds to minutes) temporal stability of implicit motor learning mechanisms. This understanding may provide a means to dissociate natural aging memory impairments from deficits caused by brain disorders that progress with aging.
\end{abstract}

Key words: aging; explicit; implicit; motor adaptation; retention; temporal

\section{Significance Statement}

Although normal aging is known to reduce learning retention, the time course and specific mechanisms that underlie these deficits are largely unknown. Here, for young and older subjects, we examined the contribution of two learning mechanisms (implicit and explicit) to motor output adjustments in response to changes in visual feedback. We also systematically quantified the short-term temporal decay of these different types of learning in both groups. We found that over a short time period explicit movement planning was not significantly different between the two age groups, but the stability of implicit motor learning was affected by normal aging. Interestingly, older subjects showed a distinctly different pattern of decay, suggesting an age-dependent impairment in the short-term retention of implicit motor learning.

Received December 4, 2020; accepted September 16, 2021; First published September 27, 2021.

The authors declare no competing financial interests.
Author contributions: W.Z. and W.M.J. designed research; G.B., R.B., R.N., and W.Z. performed research; G.B., W.Z., and W.M.J. analyzed data; G.B., W.Z., and W.M.J. wrote the paper. 


\section{Introduction}

Normal aging is consistently associated with learning and memory deficits (Seidler et al., 2010; King et al., 2013; Cai et al., 2014), which accompany impaired decision-making and motor performance (Cai et al., 2014). For example, young and older subjects can learn sequences in a serial reaction time task, but older subjects demonstrate reduced performance when learning probabilistic sequences (Dennis et al., 2006; Clark et al., 2015). In addition, younger subjects perform significantly better at pairing items in associative binding tasks (Light et al., 2004; Provyn et al., 2007; Old and Naveh-Benjamin, 2008). Aging also correlates with memory deficits (Nyberg et al., 2012; King et al., 2013); working memory declines with age (Park et al., 2002; Malouin et al., 2010), along with forms of long-term memory (e.g., episodic; Rönnlund et al., 2005; Metzler-Baddeley et al., 2011; Sauzéon et al., 2016). However, studies have also suggested that other types of memory are preserved during aging (e.g., semantic memory; Spaniol et al., 2006; St-Laurent et al., 2011; Kennedy et al., 2015).

Within the context of movement, associations between aging and reduced motor adaptation have been shown (Wolpe et al., 2020). Trewartha et al. (2014) found that despite similar adjustments to physical perturbations of arm reaching movements, older subjects demonstrated less retention and poor explicit memory performance compared with young subjects. Likewise, Malone and Bastian (2016) found that when learning a novel walking pattern, older subjects were more likely to forget and show a performance reduction after breaks. However, despite the known effect of aging on learning retention, the exact temporal properties (e.g., short-term temporal stability) of this deficit are largely unknown.

Prior studies have shown that short-term motor adaptation involves concurrent learning mechanisms with different temporal properties and drivers (i.e., error signal sensitivity; Smith et al., 2006; Taylor et al., 2014). Previous studies have also demonstrated the temporal decay of the memory trace in a wide range of tasks, suggesting that motor adaptation provides a tractable method to probe both different learning mechanisms and changes in relative stability (Sing et al., 2009; Talamini and Gorree, 2012; Hadjiosif and Smith, 2013; Kitago et al., 2013; Loaiza and McCabe, 2013; Taylor et al., 2014; Zhou et al., 2017). One notable task involves visuomotor rotation (VMR), in which individuals adapt to a visual feedback

This work was supported by the National Science Foundation (Grant 1553895 to W.M.J.) and the Alzheimer's Association (Grant AARGD-NTF-20682218 to W.M.J.).

${ }^{*}$ W.Z. and W.M.J. contributed equally to this work

Acknowledgements: We thank the participants in this study, the Davis Senior Center, and the Woodland Community \& Senior Center.

Correspondence should be addressed to Wilsaan M. Joiner at wmjoiner@ ucdavis.edu.

https://doi.org/10.1523/ENEURO.0527-20.2021

Copyright (C) 2021 Bindra et al.

This is an open-access article distributed under the terms of the Creative Commons Attribution 4.0 International license, which permits unrestricted use, distribution and reproduction in any medium provided that the original work is properly attributed. perturbation (i.e., a rotation of the feedback trajectory; Krakauer et al., 1999; Redding et al., 2005). This adaptation has been shown to involve concurrent explicit and implicit learning mechanisms (Taylor et al., 2014). Implicit learning mechanisms use sensory prediction errors to update the internal forward model, likely mediated by the cerebellum on a subconscious level (Tseng et al., 2007; Synofzik et al., 2008). Additionally, explicit processes also contribute to the adaptation to the rotation of the feedback through conscious efforts, minimizing the experienced target errors (Mazzoni and Krakauer, 2006; Bond and Taylor, 2015). Thus, the VMR paradigm provides a method for assessing both explicit and implicit components of motor adaptation; the explicit component of learning is determined through an aiming report made by the subject, while the implicit learning is based on subtracting this planned movement direction from the actual movement trajectory (Taylor et al., 2014; Morehead et al., 2015). Furthermore, studies have used this VMR task to examine age-dependent differences in learning. Bock (2005) and Buch (2003) used this paradigm to measure age-related variations in adaptation. However, these learning differences were either inferred by different methods (i.e., a combination of motor and nonmotor tests) or were based on the size of motor adaptation aftereffects. More recent studies have suggested that aging specifically reduces the extent to which explicit learning mechanisms are used during training to adjust motor output through more direct measures (Vandevoorde and Orban de Xivry, 2019) or are inferred through neuroimaging and nonmotor memory tests (Wolpe et al., 2020).

Based on the studies above, there are age-related differences in the use of concurrent learning mechanisms during motor adaptation. However, age-based variations in the short-term (seconds to minutes) temporal properties of these mechanisms (i.e., stability of the memory trace) remain uncertain. Here, we adapted a previous VMR adaptation paradigm (Hadjiosif and Smith, 2013; Zhou et al., 2017) to directly assess the extent to which normal aging affects the use of different learning mechanisms and then systematically examined their relative temporal stability over $1.5 \mathrm{~min}$. Based on the studies above, we hypothesized that older subjects would demonstrate a reduced short-term temporal stability of the overall adaptation, and we focused on quantifying the influence of age on the rate of decrease and identifying the driver (implicit or explicit learning mechanisms) of this decay process.

\section{Materials and Methods}

\section{Participants}

Twenty young subjects (10 women; age range, 19-28 years) and 19 older subjects (14 women; age range, 6385 years) without known neurologic impairment were recruited from the University of California, Davis, and the surrounding community to participate in the study. The cognitive function of all subjects was evaluated by using the Mini-Mental State Exam (MMSE; Folstein et al., 1975) and Trail Making Test (TMT, parts A and B; Army 
Individual Test Battery, 1944; Reitan and Wolfson, 1985). Scores on both the MMSE and the TMT did not reach the cutoff scores for concerns of cognitive impairment (24 of 30 on MMSE, $78 \mathrm{~s}$ for TMT part A, $273 \mathrm{~s}$ for part B) and were not significantly different between groups. Handedness of the subjects was measured by the Edinburgh Handedness Inventory (Oldfield, 1971). All young subjects recruited were right handed, and 2 of 19 older subjects were left handed. Note that performance for these two subjects was not different from that of the respective group. All subjects gave written informed consent and received financial compensation for their participation. The study protocol was approved by the University of California, Davis, Institutional Review Board.

\section{Experimental apparatus}

In the task, subjects were seated at a desk in a dimly lit room facing a horizontal 27 inch LCD monitor (Fig. 1A). The chair height was adjusted for each subject so that they could comfortably perform the task and view the screen. The experimental system included a monitor, a digitizing tablet, and a PC to run the experimental paradigm and collect the behavioral data. The LCD monitor was mounted horizontally in front of subjects at shoulder level, displaying the various visual cues during the experiment. The monitor was 10 inches above the digitizing tablet (a workspace of 12 by 19 inches; Intuos 4 , Wacom) that tracked and recorded hand position at $200 \mathrm{~Hz}$. Subjects grasped a cylindrical handle (diameter, $2.5 \mathrm{~cm}$ ) containing the tablet stylus inside. The hand/stylus moved on the tablet below the monitor (refresh rate, $60 \mathrm{~Hz}$ ), with its position presented on the above screen as a round cursor (diameter, $0.3 \mathrm{~cm}$ ). The midline of the subject was aligned with the center of the tablet and monitor. This also served as the center of the workspace. The position of the LCD monitor obstructed the vision of the tablet and the arm movements made by subjects. The large 27 inch screen provided sufficient space to ensure that the space between the edge of the screen and visual objects used in the task (e.g., the circular path of the rotation marker) did not provide unintended spatial landmarks to the subjects. A rotation knob (diameter, $3.8 \mathrm{~cm}$ ) was mounted on the left side of the tablet to allow the subjects to choose their aiming direction before each movement.

\section{Experimental paradigm}

On each trial, the subject reached from a circular central target $(8 \mathrm{~mm}$ in diameter) located at the center of the screen $(20 \mathrm{~cm}$ from the body on the sagittal axis of the body) to a circular reach target (diameter, $10 \mathrm{~mm}$ ) located $10 \mathrm{~cm}$ away from the starting position along the midline. At the start of the trial, a large circle (diameter, $10 \mathrm{~cm}$ ) was displayed centered at the starting location, showing the path for the rotation maker and indicating the spatial relationship among the marker and targets. An orange rotation marker was shown on this circular path and could be moved along the path by rotating the knob (Fig. 1B). The subject was instructed to first turn the rotation knob with their left hand to select an aiming direction for the planned movement. Once the subject had made a selection by pressing the knob, the display of the circular path and rotation maker was removed. Subjects then could initiate the movement of the cursor from the center location to the reach target using their right hand (Movie 1, VMR Decay Task.mp4, examples of the task structure/requirements and the different trials used in the experiment). Subjects were instructed to move the cursor from the start position to the target as quickly and fluidly as possible. Subjects received visual and auditory feedback on the speed of their movement to ensure consistency of speed and duration. If the movement was too slow (duration, $\geq 400 \mathrm{~ms}$ ), the target turned blue once the radial distance was exceeded. If the movement was too fast $(\leq 200 \mathrm{~ms})$, the target turned red. The target turned green for movement durations that were between 200 and 400 ms. In addition, a short-duration beep (frequency, $429 \mathrm{~Hz}$ ) was provided to signify a good trial. Note that this feedback was based only on movement duration and not on movement accuracy. On adaptation trials, during the $10 \mathrm{~cm}$ point-to-point reaching movement full visual feedback of the cursor was provided to the subject. Once the movement exceeded the radial distance of the target $(10 \mathrm{~cm})$, only the end-point feedback (cursor at a location where the movement exceeded $10 \mathrm{~cm}$ ) was provided. One second after the end-point feedback was presented, the cursor was removed and replaced by a large circle centered on the initial start location. The radius of the circle matched the distance of the hand/cursor from the start location. Subjects were guided to move their hand back to the start location by the size of the circle (the radius of the circle decreased proportionally to the distance between the hand/cursor and the start location), which limited the spatial information of the actual movements while providing enough information to guide the subject back to the start location. Once the hand/cursor was within $2 \mathrm{~cm}$ of the start location, the cursor reappeared, and the subject brought the cursor back to the central target. The intertrial interval was set to $1 \mathrm{~s}$. At the beginning of each trial, the orange rotation marker appeared $10 \mathrm{~cm}$ above the central start position along the circular path.

All subjects in both groups (young and older group) experienced the same task structure used in previous studies (Fig. 1C; Hadjiosif and Smith, 2013; Zhou et al., 2017). Subjects began the task with 20 familiarization trials to ensure that they were accustomed to the experimental design and desired movement speed. During baseline (40 trials), the cursor followed the true position of the hand/ stylus on the tablet below (Fig. 1D). On a random number of trials (20\%) visual feedback was removed (blank trials). These blank trials (a total of eight blank trials) served as the baseline for the retention probe trials described below. After baseline, subjects were trained on a visuomotor rotation (60 trials) during which the cursor path was rotated around the hand path by either a $+45^{\circ}$ [clockwise $(\mathrm{CW})]$ or $-45^{\circ}$ [counterclockwise (CCW)] perturbation. Each subject experienced only one type of rotation (CW or CCW), and the direction of the VMR was counterbalanced across all subjects. We defined three periods during training to compare learning between groups [early training: the first five training trials (1-5); middle training: 
A

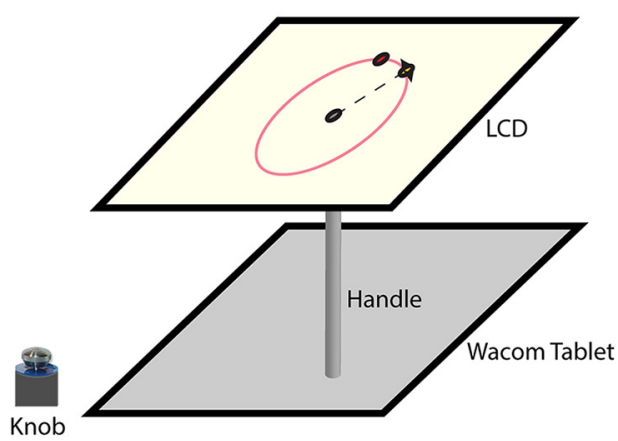

B

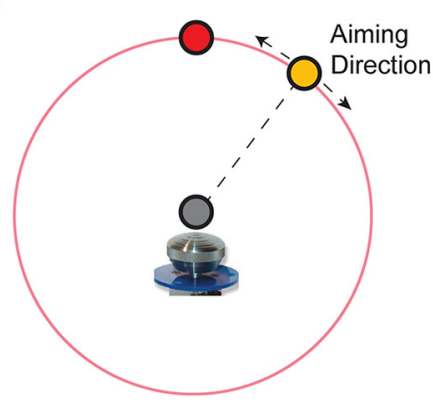

C

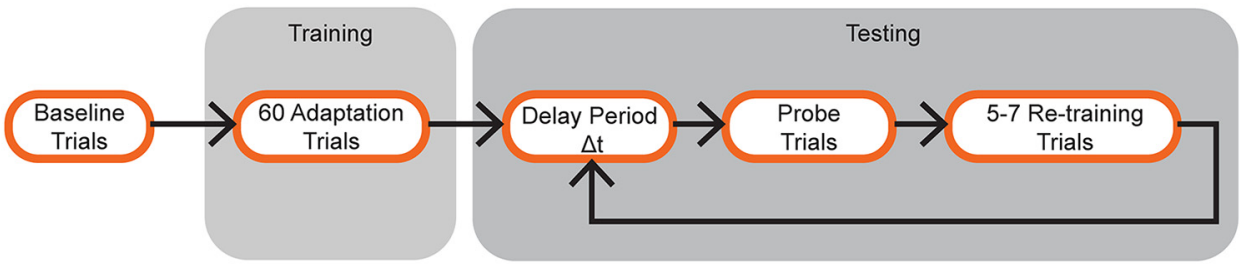

D

Trial Types

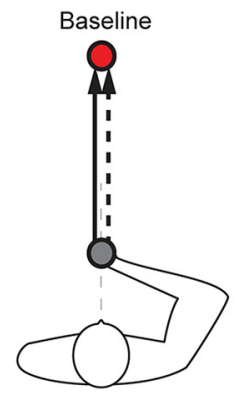

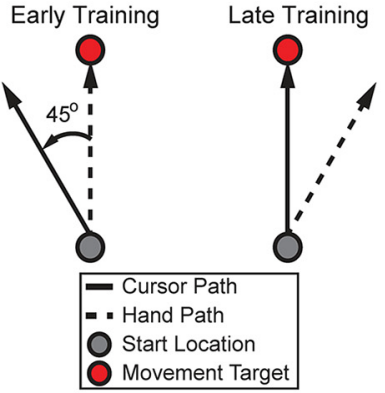

E
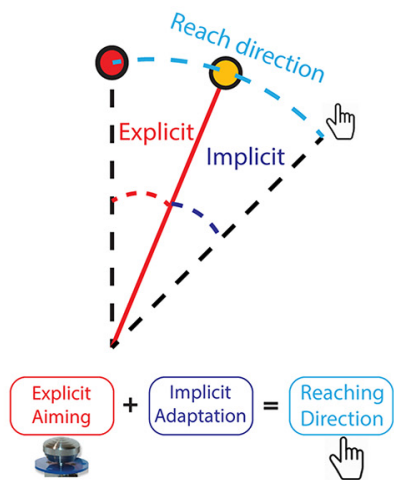

Figure 1. Experimental design and task structure. $\boldsymbol{A}$, The experimental setup consisted of an LCD monitor that displayed targets and visual feedback of the hand, a digitizing tablet, and a PC that controlled the paradigm. The monitor was 10 inches above the digitizing tablet. The midline of the subject was aligned with the center of the tablet and the monitor. B. Depiction of the explicit aim selection, which consisted of a circle centered at the start location (gray filled circle). The movement goal is represented by the red filled circle, and a yellow filled circle represents the selection marker. This circle appeared $10 \mathrm{~cm}$ from the starting position. Subjects turned a knob at the beginning of each trial to move the yellow marker around the circle and indicate their explicit aiming direction. $\boldsymbol{C}$, The sequence of training and testing the subjects. After 20 familiarization trials, the subjects underwent 40 baseline trials where the cursor followed the hand position. This was followed by 60 training trials, during which a $+45^{\circ}$ (clockwise) or $-45^{\circ}$ (counterclockwise) visuomotor rotation of the cursor movement feedback was applied. During the testing phase, each subject experienced a delay period $(0,5,10,2030,50$, and $90 \mathrm{~s})$. This was followed by a single probe trial, and then $5-7$ retraining trials before the next delay period was tested. This pattern was repeated for a total of 4 probe movements for each delay duration. $\boldsymbol{D}$, Depiction of hand and cursor movements during baseline, and early and late training. During baseline, the cursor and hand paths were aligned. When the visuomotor rotation was applied, there was a rotational offset between the cursor and hand path. Late in training, subjects adjusted the hand path to guide the cursor to the target. $\boldsymbol{E}$, A representation of the explicit and implicit components of overall adaptation. Explicit aiming was measured as the angle difference between the aiming direction and the target, while implicit adaptation was defined as the angle difference between reach direction and aiming direction.

the middle five trials of training (28-32); and late training: the last five training trials (56-60)]. Note that these periods were used to show the progression in behavior throughout training and were based on the total number of training trials. Also, the results are similar for different window sizes (three or seven trials).
After training, subjects completed the testing phase based on a task structure developed by Hadjiosif and Smith (2013). Subjects underwent a series of retention probe trials and retraining trials for seven different delay periods $(0,5,10,20,30,50$, and $90 \mathrm{~s})$. The sequence is depicted in Figure $1 \mathrm{C}$. Throughout the delay, subjects 


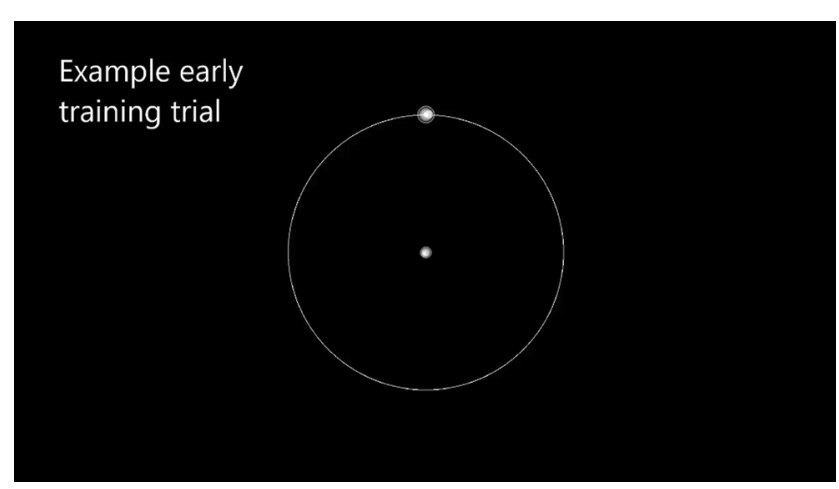

Movie 1. VMR Decay Task.mp4. The video illustrates the visual display and objects controlled during the experiment: the selection marker, the circular path, target, and cursor. Three different trial examples are sequentially shown: (1) an early training trial, (2) a late training trial, and (3) a retention probe trial (5 $s$ delay). [View online]

held the cursor in the start target, which turned light green as a signal for the subject to maintain their cursor at the center position. After the delay period, the selection marker appeared on the circular path to allow the subject to select the planned movement direction of the upcoming arm movement. The display disappeared after the selection, cueing the subject to perform the reach. This retention probe trial, which was the first trial after the delay period ended, were also blank trials, meaning that there was no visual feedback. The probe trial for each delay period was followed by five to seven retraining trials during which subjects performed movements to the trained target with the rotation and full visual feedback. This pattern (one retention probe movement followed by five to seven retraining movements) was repeated throughout the session, for a total of four probe movements for each delay duration. The delays were randomly selected throughout the session. Because of the randomness of the number of retraining trials, subjects made a total of 168-224 movements divided between two blocks. The entire session ( $40 \mathrm{~min}$ for behavioral testing and $30 \mathrm{~min}$ for clinical evaluations-the Mini-Mental State Exam and Trail Making Test) was $\sim 1 \mathrm{~h}$ and $30 \mathrm{~min}$ with breaks between blocks. A short video illustrating an example of an early training trial, a late training trial, and a retention probe trial can be found in the video VMR Decay Task.mp4 (Movie 1).

\section{Analysis of the movement data}

The movement duration was defined as the interval between the times when the hand velocity exceeded 0.05 $\mathrm{m} / \mathrm{s}$ and when the hand crossed the $10 \mathrm{~cm}$ radius. We focused on the initial heading angle of the hand-the feedforward mechanisms involved in the movement plan. We determined the reach angle of each movement soon after initiation by computing the angle of the line connecting the hand position at 1 and $3 \mathrm{~cm}$ into the movement. To quantify the contributions of explicit and implicit learning to the motor recalibration in response to the rotated visual feedback, we performed a procedure that was similar to previous studies (Taylor et al., 2014; Bond and Taylor, 2015; Morehead et al., 2015). The explicit aiming was measured by the angles that subjects selected as their aiming direction (the angle of the line connecting the start location and rotation marker). The implicit learning was calculated by subtracting the aiming angles from the actual reaching angles (Fig. 1E). For simplicity, we refer to the target direction as $0^{\circ}$ and classify all movements relative to this direction in the analysis. The experiment was designed to examine the retention of these two types of learning after short time delays. For each time delay, the retention of learning was quantified by comparing the angular deviation on the no-visual-feedback probe trials to the average angular deviation at the end of the preceding retraining trials (the last three trials of the five- to seventrial retraining sequence). The absolute retention (difference in angular deviation between the probe trial and the average on the retraining trials) and relative retention (percentage of the adaptation retained: (probe trial angle - average angle on the retraining trials)/average angle on the retraining trials) were used to examine the decay of the two learning components and overall learning over the delay periods. These measures of retention were a direct comparison of before and after the delay; there was no need for a baseline correction. Because of the variance in the selection time and movement duration across subjects, we used the actual elapsed time to define the delay for explicit and implicit learning instead of the imposed seven delay periods in the experiments to better capture the influence of the delay for each learning component. Thus, the delay for the explicit learning component was defined as the time interval from the end of the previous trial to the time when the selection was made. The delay for the implicit learning component was defined as the time interval from the end of the previous trial to the movement onset. (Note that the actual elapsed time was always slightly longer for the implicit learning component.)

As in previous work (Zhou et al., 2017), we applied a standard exponential to determine the time constant of decay, as follows:

$$
y=y_{0} \cdot e^{\frac{-t}{\tau}}+C .
$$

Here, the amount of learning $(y)$ over time, $t$, depends on (1) the scaling term, $y_{0}$; (2) the time constant, $\tau$ and (3) an offset term, $t$, that represents the amount of learning that remains stable following the short-term decay. We also fitted the data using an alternative linear model to determine which model provided a better description of the data, as follows:

$$
y=k \cdot t+C,
$$

where $k$ is the slope of the linear regression and $C$ is the intercept. We fitted the data with the two models using functions fitn/m and fit/m in MATLAB and determined the goodness-of-fit by comparing the Akaike information criterion (AIC; Akaike, 1974) of the two models (a model is considered to be a better fit for the data if its AIC is smaller than the AIC of the other model). 
In addition to determining the time course of explicit and implicit learning throughout training, and the relative stability of the memory traces following the elapsed time, we also examined the temporal properties of the selection process of the explicit movement plan. Through the rotation knob, we quantified the time required to select the explicit movement plan direction. The selection time for aiming was defined as the interval between when subjects started rotating the knob and when they pressed the knob. We examined the selection time of the explicit aiming process throughout the training trials and over the elapsed time.

\section{Statistical analysis}

Data were analyzed offline using MATLAB 2019a (MathWorks) and R version 3.6.2 (r-project.org). Trials that had very slow/fast movement speeds (peak speed, $<0.22$ or $>0.5 \mathrm{~m} / \mathrm{s}$ ) and long selection time (selection time, $>30 \mathrm{~s})$ were excluded $(\sim 3 \%$ of total trials for the young group and $\sim 4.5 \%$ of total trials for the older group). The reason we excluded the trials with a selection time $>30 \mathrm{~s}$ is that all these trials were at the beginning of the training session when subjects first experienced the visuomotor rotation and were becoming familiar with rotating the knob and making an aiming selection. There were $2.1 \%$ of training trials with a selection time $>30 \mathrm{~s}$ for the older group and $0.6 \%$ for the young group. All of these trials were in the first half of the training phase; none of the subjects made a selection with a time $>30 \mathrm{~s}$ for trials in the second half of the training session and the entire testing phase. We applied a linear mixed-effects model (LMM) in R using the ImerTest package (Kuznetsova et al., 2017) to test the fixed effects of group (young and older) and periods (different trial periods in the experiment; e.g., early learning, middle learning, and late learning periods) or time delay periods and random effect of subjects on the amount of explicit and implicit learning. To examine the potential difference in the temporal retention of the learning between the two groups, we fitted the absolute angular decay and the relative decay percentage (see above) using an LMM with fixed effects of group and learning type, a random effect of subjects, and actual elapsed time as a covariate. By considering the actual elapsed time as a continuous covariate, we were able to compare the retention between the two age groups across a continuous delay range (i.e., the short-term temporal stability of learning). The LMM models were estimated using the restricted maximum likelihood method and the significance was obtained using Kenward-Roger and Satterhwaite approximations with the pbkrtest package (Halekoh and Højsgaard, 2014). If significance was identified, post hoc tests were performed using the emmeans package (Lenth et al., 2020) and adjusted for multiple comparisons using Bonferroni-Holm corrections. Effect size (d) was calculated using Cohen's $d$ (Cohen, 1988) measurement (for LMM analysis, generalized effect size was computed following similar procedures of Cohen's $d$ measurement using the eff_size function in the emmeans package). For all tests, the significance level was set to 0.05 . In all cases, group data are presented as the mean \pm SEM. Please see the statistical table for a summary of the data structure, analysis, and confidence intervals (Cls; Table 1).

\section{Results}

\section{Baseline performance}

All subjects practiced selecting the aiming direction and reaching to the targets during the baseline block with $20 \%$ blank no-visual-feedback trials (a total of eight blank movements). During the baseline trials, all participants in both groups moved the cursor directly to the target, and the reach angles were not significantly different from each other (young: $0.45^{\circ} \pm 0.23^{\circ}$; older: $0.47^{\circ} \pm 0.29^{\circ}$; twotailed unpaired $t$ test: $p=0.97, d=0.012$ ) and not significantly different from $0^{\circ}$ (two-tailed one-sample $t$ test; young group: $p=0.16, d=0.37$; older group: $p=0.12$, $d=0.44$ ). Participants demonstrated no explicit aiming or implicit learning during this block, as expected (explicit: young, $-0.029^{\circ} \pm 0.046^{\circ}$; older, $-0.031^{\circ} \pm 0.10^{\circ}$; twotailed one-sample $t$ test; young group: $p=0.53, d=0.14$; older group: $p=0.75, d=0.073$; implicit: young, $0.48^{\circ} \pm$ $0.24^{\circ}$; older, $0.50^{\circ} \pm 0.32^{\circ}$; two-tailed one-sample $t$ test; young group: $p=0.06, d=0.45$; older group: $p=0.13$, $d=0.36$ ). Additionally, there were no significant differences between the explicit and implicit components of adaptation in both groups (an analysis using LMM with fixed effects of group and learning type and random effect of subjects: group: $F_{(1,74)}=0.0012, p=0.97$; learning type: $F_{(1,74)}=0.35, p=0.12$; interaction between the two fixed effects: $\left.F_{(1,74)}=0.0021, p=0.96\right)$.

\section{Adaptation to the visual feedback perturbation}

After the baseline period, a $45^{\circ}$ VMR (CW or CCW) was introduced for 60 trials (Fig. $2 A, B$ ). After the perturbation, the older subjects' overall learning (Fig. $2 B$, light blue trace) generally progressed in a pattern similar to that of the young subjects (Fig. 2A, light blue trace). We first evaluated the mean adaptation over the training period for both groups. Throughout the entire training period, the subjects in the young group had a significantly higher explicit learning level (young group: $17.73^{\circ} \pm 3.40^{\circ}$; older group: $11.56^{\circ} \pm 3.6^{\circ}$; two-tailed unpaired $t$ test, $p>$ $0.022, d=0.76$ ) and a similar implicit learning level compared with the subjects in the older group (young group: $16.35^{\circ} \pm 3.43^{\circ}$; older group: $18.65^{\circ} \pm 3.64^{\circ}$; two-tailed unpaired $t$ test, $p=0.26, d=0.37$ ), leading to significantly higher overall adaptation for the young group (young group: $34.07^{\circ} \pm 4.55^{\circ}$; older group: $30.20^{\circ} \pm 4.68^{\circ}$; twotailed unpaired $t$ test, $p>0.023, d=0.75$ ). To better investigate the progression of the learning and the potential difference in adaptation, we compared the learning between the two groups at three periods during training (early, middle training, and late training; see Materials and Methods). Over the entire training block, both groups demonstrated an increase in overall learning. During the three training periods, older subjects adapted at an overall rate similar to that in young subjects (early training: young group, $12.60^{\circ} \pm 2.22^{\circ}$; older group, $13.04^{\circ} \pm 1.79^{\circ}$; middle training: young group, $42.56^{\circ} \pm 2.69^{\circ}$; older group, $42.59^{\circ} \pm$ $2.65^{\circ}$; late training: young group, $46.14^{\circ} \pm 1.36^{\circ}$; older 
Table 1: Statistics table for "normal aging affects the short-term temporal stability of implicit, but not explicit, motor learning following visuomotor adaptation"

\begin{tabular}{|c|c|c|c|}
\hline Line \# & Data structure & Type of test & $95 \% \mathrm{Cl}$ \\
\hline 280 & Normal & Two-tailed unpaired $t$ test & $\begin{array}{l}\text { Baseline overall reaching angles: } \\
\text { Young: }\left[-0.026^{\circ}, 0.93^{\circ}\right] \\
\text { Older: }\left[-0.14^{\circ}, 1.08^{\circ}\right]\end{array}$ \\
\hline 281 & Normal & Two-tailed one-sample $t$ test & \\
\hline 284 & Normal & Two-tailed unpaired $t$ test & $\begin{array}{l}\text { Baseline explicit learning: } \\
\text { Young: }\left[-0.13^{\circ}, 0.068^{\circ}\right] \text {; older: }\left[-0.24^{\circ}, 0.18^{\circ}\right] \\
\text { Baseline implicit learning: } \\
\text { Young: }\left[-0.014^{\circ}, 0.98^{\circ}\right] \text {; older: }\left[-0.17^{\circ}, 1.17^{\circ}\right]\end{array}$ \\
\hline 285 & Normal & Two-tailed one-sample $t$ test & \\
\hline 288 & Normal & $\begin{array}{l}\text { Linear mixed-effects model: } \\
\text { Explicit and implicit learning during baseline } \sim \text { group } \\
\quad \times \text { learning type }+(1 \mid \text { subject })\end{array}$ & \\
\hline 312 & Normal & $\begin{array}{l}\text { Linear mixed-effects model } \\
\text { Overall learning } \sim \text { group } \times \text { training period }+(1 \mid \\
\quad \text { subject) }\end{array}$ & $\begin{array}{l}\text { Overall learning during training: } \\
\text { Young: early, }\left[7.96^{\circ}, 17.24^{\circ}\right] \text {; middle, }\left[36.92^{\circ}, 48.19^{\circ}\right] \text {; late, }\left[43.29^{\circ}, 48.99^{\circ}\right] \\
\text { Older: early, }\left[9.22^{\circ}, 16.75^{\circ}\right] \text {; middle, }\left[36.95^{\circ}, 48.12^{\circ}\right] \text {; late, }\left[40.35^{\circ}, 48.88^{\circ}\right]\end{array}$ \\
\hline 338 & Normal & $\begin{array}{l}\text { Linear mixed-effects model } \\
\text { Explicit and implicit learning during training } \sim \text { group } \\
\quad \times \text { training period } \times \text { learning type }+(1 \mid \text { subject })\end{array}$ & $\begin{array}{l}\text { Explicit learning: } \\
\text { Young: early, }\left[2.94^{\circ}, 13.12^{\circ}\right] \text {; middle, }\left[14.90^{\circ}, 26.19^{\circ}\right] \text {; late, }\left[18.23^{\circ}, 29.38^{\circ}\right] \\
\text { Older: early, }\left[0.95^{\circ}, 8.22^{\circ}\right] \text {; middle, }\left[11.58^{\circ}, 21.23^{\circ}\right] \text {; late, }\left[12.25^{\circ}, 27.88^{\circ}\right] \\
\text { Implicit learning: } \\
\text { Young: early }\left[1.29^{\circ}, 8.50^{\circ}\right] \text {; middle }\left[17.038^{\circ}, 26.98^{\circ}\right] \text {; late }\left[16.75^{\circ}, 27.92^{\circ}\right] \\
\text { Older: early, }\left[4.84^{\circ}, 11.91^{\circ}\right] \text {; middle; }\left[20.11^{\circ}, 28.19^{\circ}\right] \text {; late, }\left[18.14^{\circ}, 30.96^{\circ}\right]\end{array}$ \\
\hline 364 & Normal & Two-tailed unpaired $t$ test & $\begin{array}{l}\text { Selection time during baseline: } \\
\text { Young: }[0.66 \mathrm{~s}, 1.00 \mathrm{~s}] \\
\text { Older: }[1.02 \mathrm{~s}, 1.89 \mathrm{~s}]\end{array}$ \\
\hline 371 & Normal & $\begin{array}{l}\text { Linear mixed-effects model } \\
\text { Selection time during training } \sim \text { group } \times \text { training pe- } \\
\quad \text { riod }+(1 \mid \text { subject })\end{array}$ & $\begin{array}{l}\text { Selection time during training } \\
\text { Young: early, [3.17 s, } 6.18 \mathrm{~s}] \text {; middle, [1.80 s, } 2.66 \mathrm{~s}] \text {; late, [1.60 s, } 2.27 \mathrm{~s} \text { ] } \\
\text { Older: early, [6.11s, } 11.86 \mathrm{~s}] \text {; middle, [4.36s, 9.23s]; late, [2.92s, 6.58s] }\end{array}$ \\
\hline 432 & Normal & Two-tailed paired $t$ test & $\begin{array}{l}\text { Overall learning during retraining vs } 0 \mathrm{~s}: \\
\text { Young: retraining, }\left[44.14^{\circ}, 45.68^{\circ}\right] ; 0 \mathrm{~s},\left[44.21^{\circ}, 45.72^{\circ}\right]\end{array}$ \\
\hline 434 & Normal & Two-tailed paired $t$ test & $\begin{array}{l}\text { Overall learning during retraining vs } 0 \mathrm{~s}: \\
\text { Older: retraining, }\left[40.87^{\circ}, 43.76^{\circ}\right] ; 0 \mathrm{~s},\left[39.80^{\circ}, 43.11^{\circ}\right]\end{array}$ \\
\hline 436 & Normal & $\begin{array}{l}\text { Linear mixed-effects model } \\
\text { Absolute temporal decay of the overall adaptation } \sim \\
\quad \text { group } \times \text { actual elapsed time }+(1 \mid \text { subject })\end{array}$ & $\begin{array}{l}\text { Absolute temporal decay of the overall learning: } \\
\text { Young: } 0 \mathrm{~s},\left[-0.77^{\circ}, 0.92^{\circ}\right] ; 5 \mathrm{~s},\left[-2.96^{\circ},-0.85^{\circ}\right] ; 10 \mathrm{~s},\left[-2.45^{\circ}, 0.0192^{\circ}\right] ; 20 \mathrm{~s},\left[-3.67^{\circ},-1.04^{\circ}\right] ; 30 \mathrm{~s} \text {, } \\
\quad\left[-4.11^{\circ},-0.40^{\circ}\right] ; 50 \mathrm{~s},\left[-5.04^{\circ},-1.76^{\circ}\right] ; 90 \mathrm{~s},\left[-5.49^{\circ},-2.12^{\circ}\right] \\
\text { Older: } 0 \mathrm{~s},\left[-1.65^{\circ}, 0.99^{\circ}\right] ; 5 \mathrm{~s},\left[-2.70^{\circ},-0.86^{\circ}\right] ; 10 \mathrm{~s},\left[-3.30^{\circ},-0.66^{\circ}\right] ; 20 \mathrm{~s},\left[-5.45^{\circ},-1.59^{\circ}\right] ; 30 \mathrm{~s}, \\
\quad\left[-5.12^{\circ},-1.90^{\circ}\right] ; 50 \mathrm{~s},\left[-7.06^{\circ},-2.37^{\circ}\right] ; 90 \mathrm{~s},\left[-11.07^{\circ},-5.82^{\circ}\right]\end{array}$ \\
\hline 475 & Normal & $\begin{array}{l}\text { Linear mixed-effects model } \\
\text { Absolute temporal decay of the explicit and implicit } \\
\text { learning } \sim \text { group } \times \text { actual elapsed time } \times \text { learning } \\
\text { type }+(1 \mid \text { subject })\end{array}$ & $\begin{array}{l}\text { Absolute temporal decay of the explicit learning: } \\
\text { Young:\& } 0 \mathrm{~s},\left[-0.89^{\circ}, 0.83^{\circ}\right] ; 5 \mathrm{~s},\left[-0.61^{\circ}, 1.02^{\circ}\right] ; 10 \mathrm{~s},\left[-0.78^{\circ}, 1.06^{\circ}\right] ; 20 \mathrm{~s},\left[-1.07^{\circ}, 0.41^{\circ}\right] ; 30 \mathrm{~s},\left[-0.78^{\circ} \text {, }\right. \\
\left.\quad 0.84^{\circ}\right] ; 50 \mathrm{~s},\left[-0.30^{\circ}, 0.76^{\circ}\right] ; 90 \mathrm{~s},\left[-0.99^{\circ}, 0.94^{\circ}\right] \\
\text { Older: } 0 \mathrm{~s},\left[-0.63^{\circ}, 0.16^{\circ}\right] ; 5 \mathrm{~s},\left[-1.02^{\circ}, 0.18^{\circ}\right] ; 10 \mathrm{~s},\left[-0.63^{\circ}, 0.22^{\circ}\right] ; 20 \mathrm{~s},\left[-1.08^{\circ}, 0.51^{\circ}\right] ; 30 \mathrm{~s},\left[-0.58^{\circ} \text {, }\right. \\
\left.\quad 0.57^{\circ}\right] ; 50 \mathrm{~s},\left[-0.96^{\circ}, 0.30^{\circ}\right] ; 90 \mathrm{~s},\left[-0.84^{\circ}, 0.58^{\circ}\right] \\
\text { Absolute temporal decay of the implicit learning: } \\
\text { Young: } 0 \mathrm{~s},\left[-1.23^{\circ}, 1.14^{\circ}\right] ; 5 \mathrm{~s},\left[-2.92^{\circ},-0.13^{\circ}\right] ; 10 \mathrm{~s},\left[-2.64^{\circ}, 0.63^{\circ}\right] ; 20 \mathrm{~s},\left[-4.61^{\circ},-0.75^{\circ}\right] ; 30 \mathrm{~s}, \\
\quad\left[-3.74^{\circ},-0.25^{\circ}\right] ; 50 \mathrm{~s},\left[-4.63^{\circ},-1.85^{\circ}\right] ; 90 \mathrm{~s},\left[-5.70^{\circ},-1.44^{\circ}\right] \\
\text { Older: } 0 \mathrm{~s},\left[-1.48^{\circ}, 1.14^{\circ}\right] ; 5 \mathrm{~s},\left[-1.97^{\circ}, 0.36^{\circ}\right] ; 10 \mathrm{~s},\left[-2.78^{\circ}, 0.32^{\circ}\right] ; 20 \mathrm{~s},\left[-4.84^{\circ},-1.48^{\circ}\right] ; 30 \mathrm{~s},\left[-5.81^{\circ},\right. \\
\left.\quad-1.66^{\circ}\right] ; 50 \mathrm{~s},\left[-5.77^{\circ},-1.61^{\circ}\right] ; 90 \mathrm{~s},\left[-9.83^{\circ},-6.12^{\circ}\right]\end{array}$ \\
\hline 504 & Normal & $\begin{array}{l}\text { Linear mixed-effects model } \\
\text { Relative temporal decay of the explicit and implicit } \\
\text { learning } \sim \text { group } \times \text { actual elapsed time } \times \text { learning } \\
\text { type }+(1 \mid \text { subject })\end{array}$ & $\begin{array}{l}\text { Relative temporal decay of the explicit learning: } \\
\text { Young: } 0 \mathrm{~s},[-2.98 \%, 4.02 \%] ; 5 \mathrm{~s},[-1.69 \%, 5.54 \%] ; 10 \mathrm{~s},[-3.13 \%, 3.67 \%] ; 20 \mathrm{~s},[-2.67 \%, 2.63 \%] ; 30 \mathrm{~s} \text {, } \\
\quad[-3.86 \%, 2.81 \%] ; 50 \mathrm{~s},[-1.50 \%, 4.32 \%] ; 90 \mathrm{~s},[-3.40 \%, 3.79 \%] \\
\text { Older: } 0 \mathrm{~s},[-3.71 \%, 3.38 \%] ; 5 \mathrm{~s},[-9.34 \%, 2.55 \%] ; 10 \mathrm{~s},[-4.21 \%, 1.75 \%] ; 20 \mathrm{~s},[-6.26 \%, 4.34 \%] ; 30 \mathrm{~s} \text {, } \\
\quad[-4.66,9.25 \%] ; 50 \mathrm{~s},[-9.89 \%, 4.22 \%] ; 90 \mathrm{~s},[-4.82 \%, 5.19 \%] \\
\text { Relative temporal decay of the implicit learning: } \\
\text { Young: } 0 \mathrm{~s},[-8.37 \%, 5.45 \%] ; 5 \mathrm{~s},[-16.04 \%,-1.63 \%] ; 10 \mathrm{~s},[-14.04 \%, 0.24 \%] ; 20 \mathrm{~s},[-19.36 \%, \\
\quad-5.20 \%] ; 30 \mathrm{~s},[-19.03,-1.50 \%] ; 50 \mathrm{~s},[-21.68 \%,-7.82 \%] ; 90 \mathrm{~s},[-23.88 \%,-6.41 \%] \\
\text { Older: } 0 \mathrm{~s},[-12.49 \%, 3.40 \%] ; 5 \mathrm{~s},[-6.78 \%, 3.03 \%] ; 10 \mathrm{~s},[-14.86 \%,-0.92 \%] ; 20 \mathrm{~s},[-19.27 \%, \\
\quad-1.83 \%] ; 30 \mathrm{~s},[-19.10 \%,-3.03 \%] ; 50 \mathrm{~s},[-28.13 \%,-9.73 \%] ; 90 \mathrm{~s},[-37.36 \%,-15.57 \%]\end{array}$ \\
\hline 518 & Normal & Two-tailed paired $t$ test & $\begin{array}{l}\text { Selection time during retraining vs. end of training } \\
\text { Young: retraining, [1.34 s, } 1.93 \mathrm{~s}] \text {; end of training }[1.60 \mathrm{~s}, 2.27 \mathrm{~s}]\end{array}$ \\
\hline 519 & Normal & Two-tailed paired $t$ test & $\begin{array}{l}\text { Selection time during retraining vs end of training } \\
\text { Older: retraining, [2.30 s, } 5.19 \mathrm{~s}] \text {; end of training, [2.92 s, } 6.58 \mathrm{~s}] \\
\quad \text { (Continued) }\end{array}$ \\
\hline
\end{tabular}


Table 1: Continued

\begin{tabular}{|c|c|c|c|}
\hline Line \# & Data structure & Type of test & $95 \% \mathrm{Cl}$ \\
\hline \multirow[t]{3}{*}{521} & Normal & Linear mixed-effects model & Selection time during adaptation decay: \\
\hline & & $\begin{array}{l}\text { Selection time during adaptation decay } \sim \text { group } \times \\
\text { delay period }+(1 \mid \text { subject })\end{array}$ & $\begin{array}{l}\text { Young: } 0 \mathrm{~s},[1.68 \mathrm{~s}, 2.65 \mathrm{~s}] ; 5 \mathrm{~s},[2.14 \mathrm{~s}, 3.05 \mathrm{~s}] ; 10 \mathrm{~s},[2.28 \mathrm{~s}, 3.31 \mathrm{~s}] ; 20 \mathrm{~s},[2.40 \mathrm{~s}, 3.33 \mathrm{~s}] ; 30 \mathrm{~s},[2.48 \mathrm{~s} \\
\quad 4.06 \mathrm{~s}] ; 50 \mathrm{~s}[2.66 \mathrm{~s}, 3.98 \mathrm{~s}] ; 90 \mathrm{~s}[2.68 \mathrm{~s}, 3.77 \mathrm{~s}]\end{array}$ \\
\hline & & & $\begin{array}{l}\text { Older: } 0 \mathrm{~s},[2.40 \mathrm{~s}, 4.87 \mathrm{~s}] ; 5 \mathrm{~s}[3.63 \mathrm{~s}, 6.53 \mathrm{~s}] ; 10 \mathrm{~s}[4.04 \mathrm{~s}, 6.53 \mathrm{~s}] ; 20 \mathrm{~s}[4.73 \mathrm{~s}, 8.25 \mathrm{~s}] ; 30 \mathrm{~s}[4.82 \mathrm{~s}, 8.43 \\
\text { s]; } 50 \mathrm{~s}[4.31 \mathrm{~s}, 7.19 \mathrm{~s}] ; 90 \mathrm{~s}[4.74 \mathrm{~s}, 8.26 \mathrm{~s}]\end{array}$ \\
\hline \multirow[t]{3}{*}{535} & Normal & Two-tailed unpaired $t$ test & Selection time during retraining \\
\hline & & & Young: $[1.34 \mathrm{~s}, 1.93 \mathrm{~s}]$ \\
\hline & & & Older: $[2.30 \mathrm{~s}, 5.19 \mathrm{~s}]$ \\
\hline \multirow[t]{3}{*}{541} & Normal & Linear mixed-effects model & Change of selection time during adaptation decay: \\
\hline & & $\begin{array}{l}\text { Change of selection time during adaptation decay } \sim \\
\text { group } \times \text { delay period }+(1 \mid \text { subject })\end{array}$ & $\begin{array}{l}\text { Young: } 0 \mathrm{~s},[0.27 \mathrm{~s}, 0.80 \mathrm{~s}] ; 5 \mathrm{~s}[0.68 \mathrm{~s}, 1.15 \mathrm{~s}] ; 10 \mathrm{~s},[0.83 \mathrm{~s}, 1.52 \mathrm{~s}] ; 20 \mathrm{~s},[0.93 \mathrm{~s}, 1.53 \mathrm{~s}] ; 30 \mathrm{~s},[1.00 \mathrm{~s} \\
2.32 \mathrm{~s}] ; 50 \mathrm{~s},[1.23 \mathrm{~s}, 2.10 \mathrm{~s}] ; 90 \mathrm{~s},[1.25 \mathrm{~s}, 2.02 \mathrm{~s}]\end{array}$ \\
\hline & & & $\begin{array}{l}\text { Older: } 0 \mathrm{~s},[-0.59 \mathrm{~s}, 1.17 \mathrm{~s}] ; 5 \mathrm{~s},[-0.05 \mathrm{~s}, 2.87 \mathrm{~s}] ; 10 \mathrm{~s},[0.48 \mathrm{~s}, 2.59 \mathrm{~s}] ; 20 \mathrm{~s},[1.96 \mathrm{~s}, 4.41 \mathrm{~s}] ; 30 \mathrm{~s},[1.97 \mathrm{~s} \\
\quad 4.47 \mathrm{~s}] ; 50 \mathrm{~s},[1.60 \mathrm{~s}, 3.45 \mathrm{~s}] ; 90 \mathrm{~s},[1.97 \mathrm{~s}, 4.59 \mathrm{~s}]\end{array}$ \\
\hline
\end{tabular}

group, $44.67^{\circ} \pm 2.01^{\circ}$ ). An LMM analysis with a random effect of subjects was used to examine two fixed effects of group (young and older) and training period (early, middle, and late training) on overall learning. This showed that training period had a significant effect on overall learning $\left(F_{(2,74)}=185.61, p<0.001\right)$, but there was not a significant effect of group $\left(F_{(1,37)}=0.032, p=0.85\right)$ or an interaction between the two fixed effects $\left(F_{(2,74)}=0.144, p=0.86\right)$. Post hoc tests showed that subjects in both groups adapted to the VMR perturbation rapidly and their overall learning during the middle training period was significantly greater than the learning during the early training period (young group: $p<0.001, d=3.62$; older group, $p<0.001$, $d=3.57$ ). All subjects almost reached their asymptotic performance during the middle training period, and their learning did not increase significantly between the middle and late training periods (young group, $p=0.95, d=0.43$; older group, $p=0.52, d=0.25$ ).

Despite similarities in the overall adaptation, the subject groups varied in their patterns of explicit and implicit adaptation (Fig. $2 A$, red trace, $B$, dark blue trace). During early training, the young subjects (Fig. $3 A$ ) demonstrated a greater increase in their explicit aiming than in their implicit learning (explicit, $8.03^{\circ} \pm 2.43^{\circ}$; implicit, $4.89^{\circ} \pm$ $\left.1.72^{\circ}\right)$. The explicit and implicit learning curves for the young subjects both reached an asymptote in the middle of the training block at similar levels (explicit, $20.55^{\circ} \pm$ $2.70^{\circ}$; implicit, $22.01^{\circ} \pm 2.38^{\circ}$ ), and both explicit and implicit learning components remained at similar levels at the end of the training (explicit, $23.80^{\circ} \pm 2.66^{\circ}$; implicit, $\left.22.34^{\circ} \pm 2.67^{\circ}\right)$. This pattern differed from that in the older subjects (Fig. $3 B$ ), in that during early training the explicit and implicit learning components had the opposite relationship compared with young subjects (explicit, $4.58^{\circ} \pm 1.73^{\circ}$; implicit, $8.41^{\circ} \pm 1.69^{\circ}$ ). In the middle training period, implicit learning remained greater than explicit aiming (explicit, $16.42^{\circ} \pm 2.22^{\circ}$; implicit, $24.19^{\circ} \pm 1.87^{\circ}$ ). Similar to the young subjects, as training progressed for the older subjects, the two learning components converged to similar levels at the end of the training session (explicit, $20.08^{\circ} \pm 3.71^{\circ}$; implicit, $24.58^{\circ} \pm 3.06^{\circ}$ ). An LMM with random effects of subjects was used to examine the fixed effects of group, learning type, and training period on the learning levels acquired by subjects in both groups. We found that there was a significant main effect of training period $\left(F_{(1,220)}=51.05, p<0.001\right)$ and an interaction between group and learning type $\left(F_{(1,220)}=4.94, p=0.028\right)$. However, there were no other significant interactions among the three fixed effects (interaction between training period and group: $F_{(2,220)}=0.046, p=0.95$; interaction between training period and learning type: $F_{(2,220)}=0.78, p=0.46$; interaction among the three fixed effects: $F_{(2,220)}=0.010$, $p=0.98)$. Post hoc tests showed a significant increase in both explicit and implicit learning components from early training to middle training for both groups $(p<0.0038$, $d>1.08$ for all cases), with no significant change beyond the middle training period (all cases: $p>0.93, d<0.33$ ). During early and late training, explicit and implicit learning components were not significantly different for both groups (young subjects: early, $p=0.36, d=0.29$; late, $p=0.67$, $d=0.13$; older subjects: early, $p=0.28, d=0.35$; late, $p=$ $0.20, d=0.41$ ). However, during middle training, there was a significant difference between the explicit and implicit components for older subjects $(p=0.035, d=0.71)$, while the difference was not significant for younger subjects $(p=0.67, d=0.13)$. The two learning components were also compared across the two age groups in the post hoc tests. We found that for all training periods, subjects in the two groups applied similar levels of explicit aiming (three training periods: $p>0.24, d<0.38$ ) and implicit learning (all three training periods: $p>0.32, d<0.31$ ) to counter the perturbation.

\section{Selection time of explicit aim direction during training}

We also compared the selection time for the direction report (see Materials and Methods) between the two groups during training (Fig. $4 A, B$ ). For the eight baseline trials, we observed that older subjects required significantly more selection time, although the VMR was not applied (young, $0.87 \pm 0.10 \mathrm{~s}$; older, $1.46 \pm 0.21 \mathrm{~s}$; twotailed unpaired $t$ test: $p=0.015, d=0.82$ ). This indicates that older subjects initially required a longer time to operate the knob. Interestingly, both groups required a large selection time to choose their aiming directions after the first exposure to the VMR in early training (young, $4.68 \pm$ $0.72 \mathrm{~s}$; older, $9.00 \pm 1.37 \mathrm{~s}$ ). For both groups, the changes in the selection time followed a similar pattern as subjects learned the task, with an initial sharp increase and 
A

- Explicit
- Implicit
- Overall

A

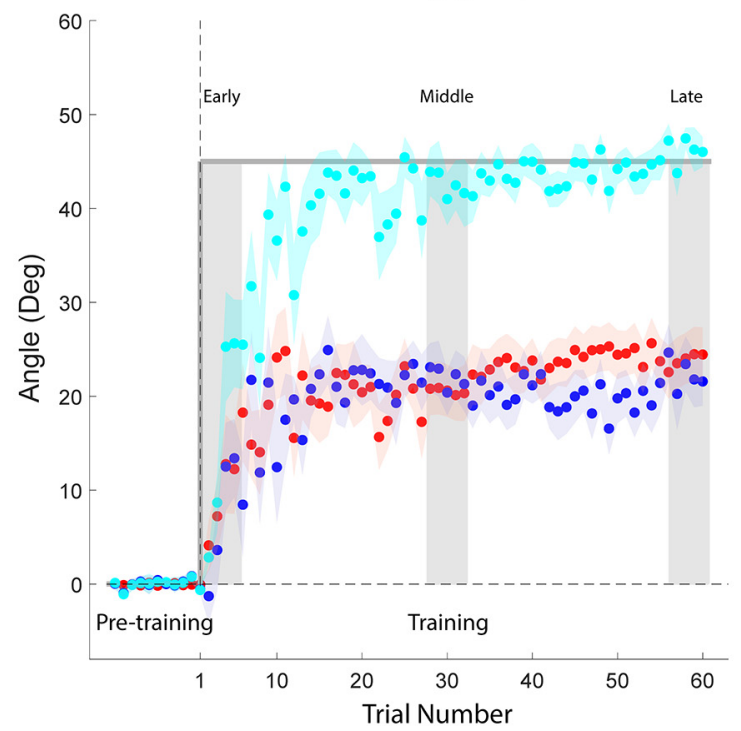

B

Adaptation over training period Older group

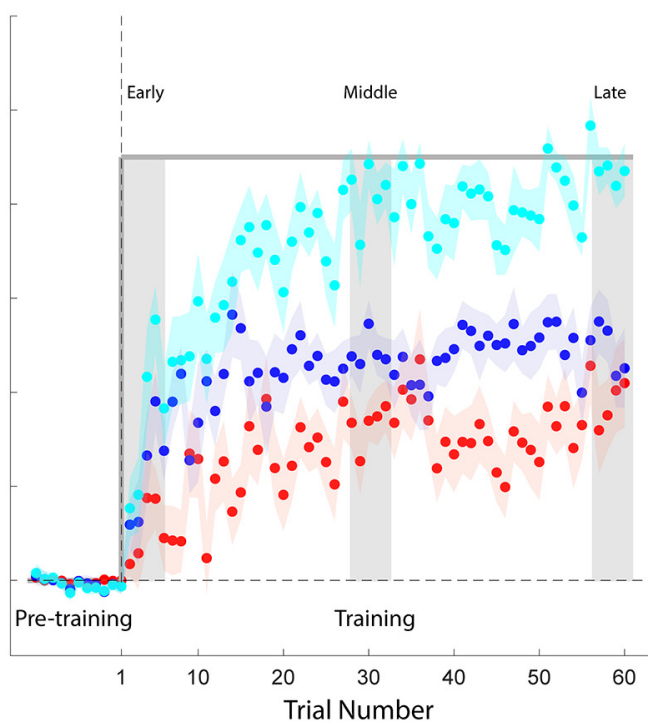

Figure 2. Adaptation curves over the training period. $\boldsymbol{A}, \boldsymbol{B}$, The learning curves for young $(\boldsymbol{A})$ and older $(\boldsymbol{B})$ subjects during training. The $x$-axis represents the progression of trials, divided into pretraining (baseline, without the visuomotor rotation perturbation) and training (which was composed of 60 trials). The dark gray trace represents the magnitude of the rotation of visual feedback, and light gray vertical bars are the three periods during training (early: the first five trials; middle: the middle five trials; and late: the last five trials). Overall and implicit adaptation, and explicit aiming levels were measured in units of degrees, through the methods depicted by Figure $1 E$. The light blue symbols represent the mean overall adaptation on each trial, while red and dark blue symbols represent the mean explicit and implicit adaptation levels on each trial, respectively. Background shading represents the SEM.
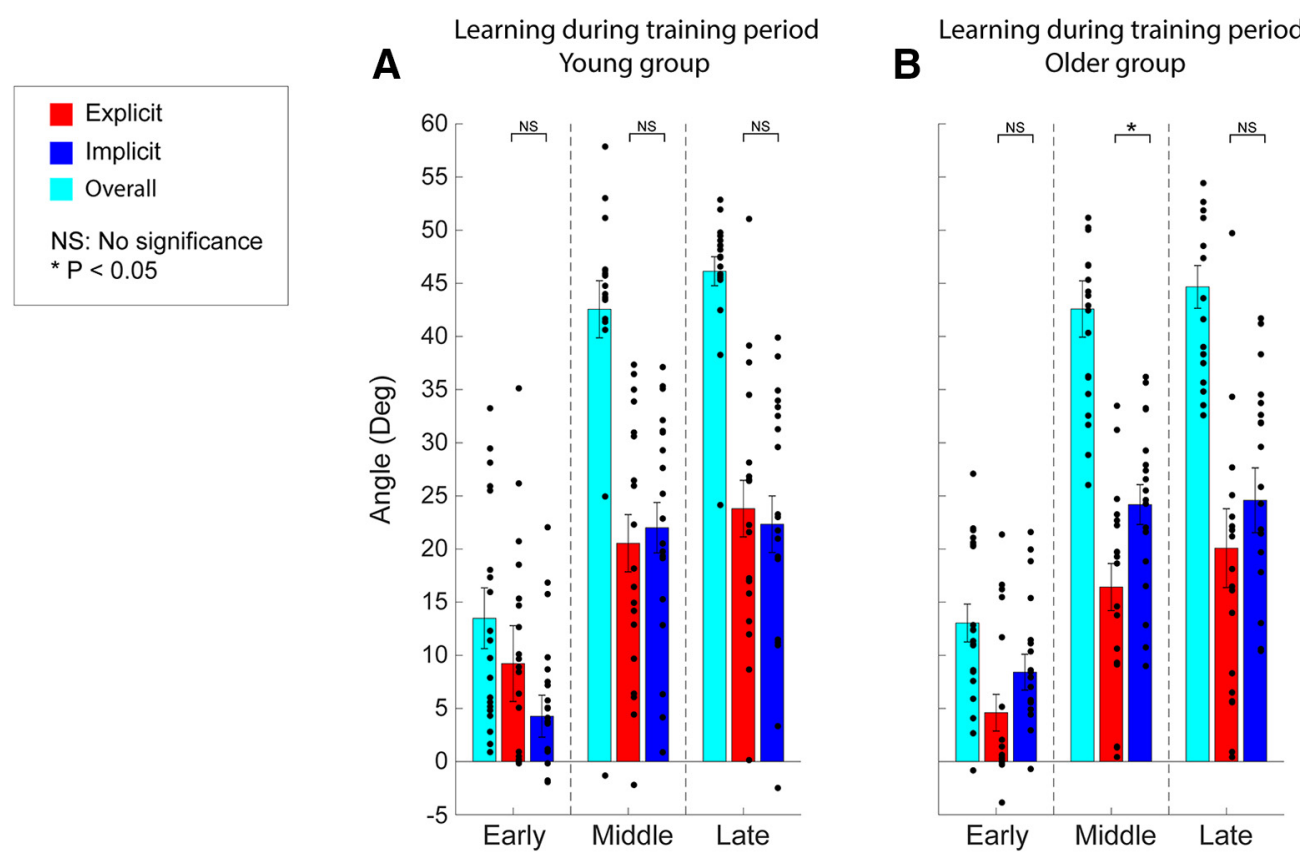

Figure 3. Overall adaptation, and levels of explicit and implicit learning during training. $\boldsymbol{A}, \boldsymbol{B}$, Histograms of the average amount of explicit, implicit, and overall adaptation that occurred for young $(\boldsymbol{A})$ and older $(\boldsymbol{B})$ subject groups during the early, middle, and late training periods. Overall, explicit, and implicit adaptation were measured in degrees, as described in Figure $1 E$. The light blue symbols represent the overall adaptation, while red and dark blue symbols represent the explicit and implicit adaptation levels, respectively. The individual data for each subject are represented by the black filled circles next to the respective histogram. Vertical error bars represent the SEM. 


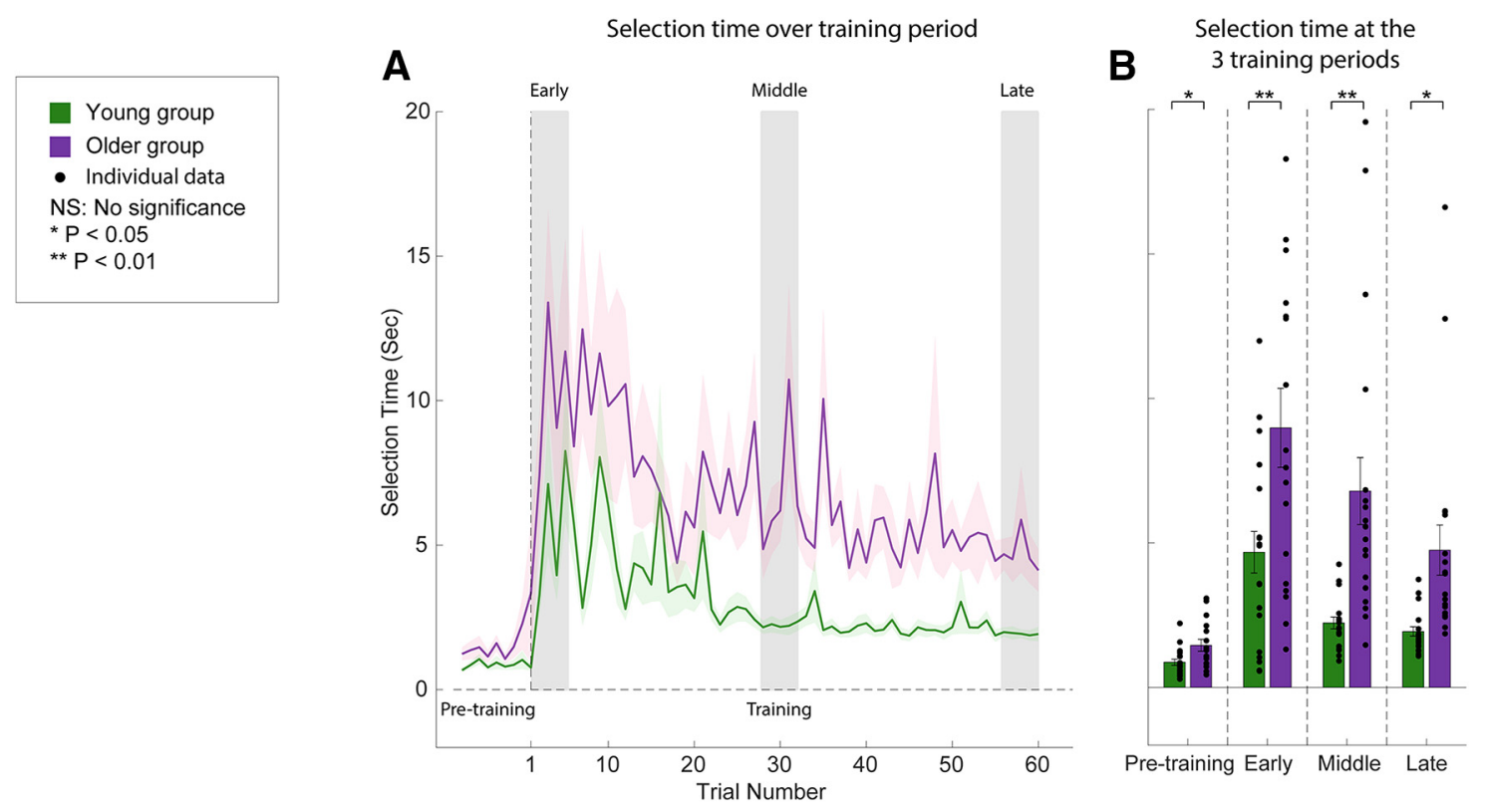

Figure 4. Explicit movement direction selection time over the training period. $\boldsymbol{A}$, Selection time was plotted as a function of trial number during pretraining (baseline, without the visuomotor rotation perturbation) and training period (which was composed of 60 trials). The young and older subject groups are represented by green and purple traces, respectively. $\boldsymbol{B}$, Histograms of the selection time at the three training periods (early, middle, and late) for young and older subjects. The individual data for each subject are represented by the black filled circles next to the respective histogram. Vertical error bars represent the SEM.

reaching an asymptote at the end of the training, albeit at different levels (late training: young, $1.94 \pm 0.16 \mathrm{~s}$; older, $4.75 \pm 0.87 \mathrm{~s}$ ). An LMM with random effect of subjects was used to test the fixed effects of group and training period (early, middle, and late) on the selection time during training. We found that both effects were significant (main effect of group: $F_{(1,74)}=10.69, p<0.001$; main effect of training period: $\left.F_{(2,37)}=22.42, p<0.001\right)$ with no interaction between them $\left(F_{(2,74)}=0.76, p=0.47\right)$. This was also the case when the initial baseline differences were taken into account. Post hoc tests showed that the selection time for subjects in both groups significantly decreased at the end of training compared with early training (young group: $p=0.038, d=0.81$; older group, $p=0.001, d=$ 1.25). For all training periods, older subjects required a significantly longer selection time than young subjects (Fig. 4B; training periods: $p<0.022, d>0.83$ ). Thus, at the end of training, although subjects in the two groups fully learned the VMR and adopted similar levels of explicit strategy to compensate for the perturbation (Figs. 2, 3), older subjects still required a significantly longer selection than young subjects.

To further examine the relationship between explicit aiming and selection time, we plotted the former as a function of the latter in Figure 5. As subjects began to develop an explicit aiming strategy during early training (Fig. 5, top row), the major axes of the ellipses were oriented toward a positive slope for both age groups, indicating that the explicit aiming directions increased as subjects used a longer selection time. After further adaptation during the middle and late training periods (Fig. 5, middle and bottom rows, respectively), the confidence ellipses for both groups rotated counterclockwise and their major axes oriented toward similar directions along the $y$ axis $\left(90^{\circ}\right)$. This change in orientation is reflected in Figures 2 and 4; over the training period, subjects in the respective groups selected similar aiming directions (Fig. 2, asymptotes, red traces) within a shorter time (Fig. 4, asymptotes, purple and green traces). However, as noted above, older subjects still required a longer selection time than the young subjects, which is demonstrated by the wider minor axes of the corresponding confidence ellipses. To quantify the relationship between explicit aiming and selection time, we applied a model type II linear regression (using the Imodel2 package in $\mathrm{R}$; Legendre, 2018) on the two variables (because both explicit aiming and selection time are noisy measurements, we used model II linear regression instead of model I). The black solid lines in Figure 5 are the model II regression fit, and the black dashed lines are the $95 \%$ Cls for the regression. During the early training period, the slopes for the linear regressions were significant for both groups (young subjects: 5.92; 95\% Cl, [3.93, 8.92]; $p<0.02$; older subjects: 1.72; $95 \% \mathrm{Cl},[1.13,2.62] ; p<0.05)$. During the middle and late training periods, the slopes of the linear regression became steeper for both groups showing that subjects were able to use a shorter time to select their aiming directions (middle training: young subjects, $-11.32 ; 95 \% \mathrm{Cl},[-18.02,-7.11] ; p=0.12$; older subjects, $3.60 ; 95 \% \mathrm{Cl}$, $[2.16,5.97] ; p=0.42$; late training: young subjects, $-17.81 ; 95 \% \mathrm{Cl},[-28.63,-11.08]$; $p=0.40$; older subjects, $5.64 ; 95 \% \mathrm{Cl}$, [3.55, 8.97]; $p=0.11$ ). Furthermore, the slopes of the regression were not significant for both groups during middle and late training, indicating that across subjects the explicit aiming strategy used to compensate for the visuomotor rotation did not significantly change with selection time. 
- Young group

- Older group

$1095 \%$ confidence ellipse

- Model II linear regression $95 \%$ confidence interval

Explicit aiming vs. Selection time

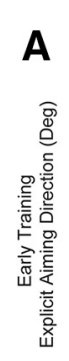

A Young gorup
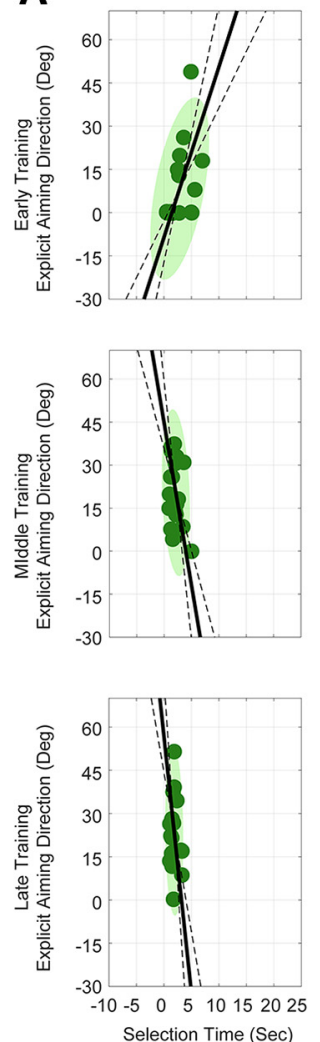

Explicit aiming vs. Selection time

B Older gorup
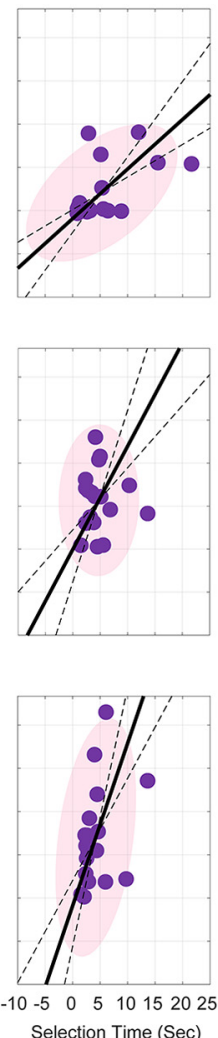

Figure 5. Relationship between explicit aiming direction and selection time during adaptation. $\boldsymbol{A}, \boldsymbol{B}$, Explicit aiming directions during the three training periods (early, middle, and late) as a function of selection time for young $(\boldsymbol{A})$ and older $(\boldsymbol{B})$ subjects. The young and older subject groups are represented by green and purple symbols, respectively. Each filled circle represents the mean explicit aiming direction and the corresponding selection time during each training period for each subject. The shaded ellipses are the $95 \%$ confidence ellipses of the data. The black solid lines represent model type II linear regression fits to the data, and the black dashed lines represented the $95 \%$ confidence intervals for the regression.

\section{Temporal decay of implicit and explicit learning}

We first assessed the retention of explicit aiming, and implicit and overall learning by comparing the aiming angles and reaching angles on the nonvisual feedback probe trials to the average aiming and reaching angles over the last three retraining trials (see Materials and Methods). We plotted the former as a function of the latter for each individual probe trial in Figure 6. For the explicit learning (Fig. $6 A$ ), the majority of the data points for both groups were on the diagonal unity lines for the different delay periods, indicating no obvious decay over time (note the mean values in each panel of Fig. 6 are on the unity lines). For the implicit learning and overall learning (Fig. 6B,C), most data points started to fall below the unity line as the delay period increased. Also, the data points for the older group (Fig. 6B,C, purple squares) deviated further from the diagonal unity lines than those for the young group (Fig. 6B,C, green circles), suggesting greater decay with time (Fig. $6 B, C$, the mean values are below the unity line, with the data for the older group below the young).

To better observe the difference between groups and to quantify the retention of learning, absolute angular decay (Fig. 7A) and relative learning decay (Fig. 7B) for the probe trials compared with the end of the preceding retraining trials were examined (see Materials and Methods). For angular decay (Fig. $7 A$ ), at the delay of $0 \mathrm{~s}$, the amount of compensation for the VMR was not significantly different from the amount of overall recalibration during retraining for both groups (young group: retraining, $44.94^{\circ}$ $\pm 0.66^{\circ} ; 0 \mathrm{~s}, 44.97^{\circ} \pm 0.81^{\circ}$; two-tailed paired $t$ test, $p>0.94, d=0.0065$; older group: retraining, $42.29^{\circ} \pm$ $0.91^{\circ} ; 0 \mathrm{~s}, 41.47^{\circ} \pm 1.22^{\circ}$; two-tailed paired $t$ test, $p>0.48, d=0.17)$. To investigate the difference in temporal decay of overall adaptation (reaching angles) between the two groups, we fitted the angular decay data using an LMM with fixed effects of group, random effect of subjects, and actual elapsed time as a covariate. Throughout the delay periods (range, 0-90 s), the temporal decay of the overall adaptation for young subjects (Fig. $6 A$, light blue circles) was consistent with previous studies (Hadjiosif and Smith, 2013; Zhou et al., 2017). There were significant effects of group and delay period on the retention of overall learning (main effect of group: $F_{(1,51.68)}=10.55$, $p=0.002$; main effect of delay period: $F_{(1,215.34)}=85.73$, $p=0.0001$ ), and the interaction between the two effects was also significant $\left(F_{(1,215.34)}=13.60, p=0.0003\right)$. Thus, the overall temporal retention for both groups decayed significantly 

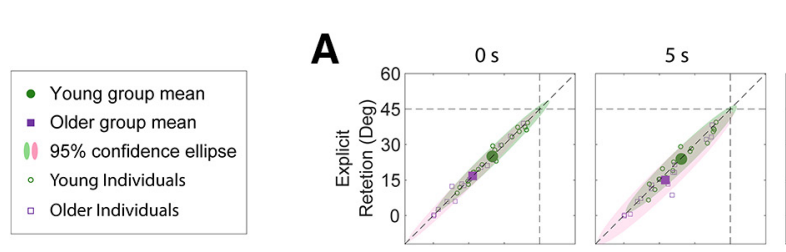

Retention evels compared to the preceding re-training trials

B
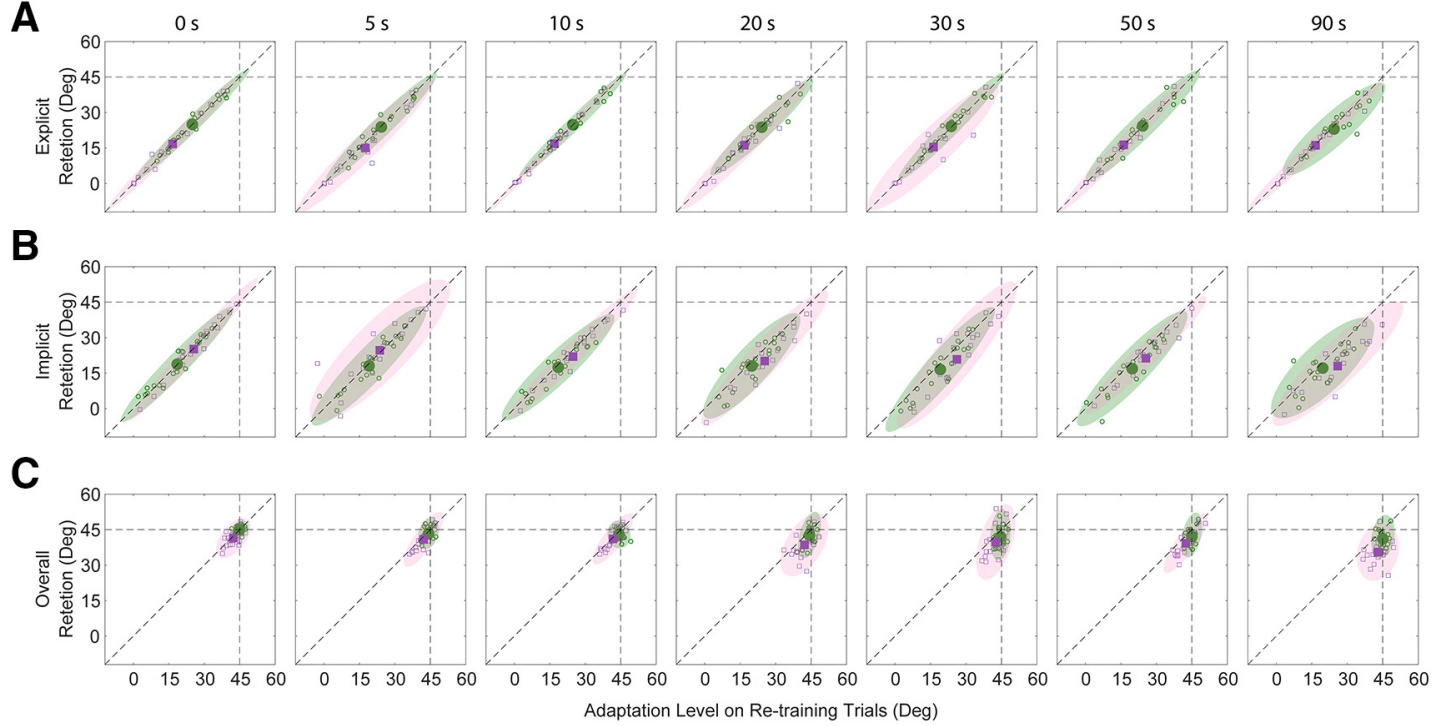

Figure 6. Retention of learning with respect to previous retraining trials. $\boldsymbol{A}-\boldsymbol{C}$, The amount of learning (aiming and reaching angle) on the probe trial is plotted against the amount of learning on the previous retraining trials for explicit aiming $(\boldsymbol{A})$, implicit learning $(\boldsymbol{B})$, and overall learning $(\boldsymbol{C})$ for the seven different delays (each respective column). The individual data for each subject are represented by the green circles (young subjects) and purple squares (older subjects). The group mean in each panel is depicted by the larger filled symbols for each respective group, and the shaded ellipses represent the $95 \%$ confidence intervals. Horizontal and vertical dashed lines show the maximum VMR perturbation $\left(45^{\circ}\right)$, and the diagonal dashed line is the unity line.

as the actual delay period increased (values are actual delay and mean delay period: young group: $0 \mathrm{~s}, 2.47 \pm 0.19 \mathrm{~s} ; 5 \mathrm{~s}$, $8.01 \pm 0.23 \mathrm{~s} ; 10 \mathrm{~s}, 13.19 \pm 0.27 \mathrm{~s} ; 20 \mathrm{~s}, 23.11 \pm 0.30 \mathrm{~s} ; 30$ s, $33.32 \pm 0.24$ s; 50 s, $53.71 \pm 0.33$ s; 90 s, $93.07 \pm 0.65 \mathrm{~s}$; older group: $0 \mathrm{~s}, 4.01 \pm 0.52 \mathrm{~s} ; 5 \mathrm{~s}, 10.55 \pm 0.81 \mathrm{~s} ; 10 \mathrm{~s}$, $16.14 \pm 0.75$ s; 20 s, $26.74 \pm 0.88$ s; 30 s, $36.81 \pm 0.86$ s; 50 s, $55.16 \pm 0.1 .19 \mathrm{~s} ; 90 \mathrm{~s}, 96.91 \pm 0.96 \mathrm{~s})$. The decay of the older group was significantly larger than the decay of the young group over time. We fit the decay data with both the exponential and linear decay models (see "Materials and Methods") and found that the pattern of temporal decay of overall learning for young subjects was well represented by the single exponential decay model (Fig. 6A, light blue line; exponential model: $R^{2}=0.54, p=0.00,011$, AIC =627.13; vs linear model: $\left.R^{2}=0.45, p=0.00,013, \mathrm{AIC}=648.20\right)$. The time constant of the exponential fit was $25.61 \pm 23.08 \mathrm{~s}$, indicating that only a portion of the overall learning was dependent on the passage of time. In contrast to the young subjects, the temporal decay of overall learning for the older group was best described by the linear model (Fig. 6B, light blue line; exponential model: $R^{2}=0.42, p<$ 0.0001 , AIC $=650.39$; vs linear model: $R^{2}=0.46, p<$ $0.0001, A I C=641.56)$. The slope of the linear model $k$ was $-0.079 \pm 0.011$. Thus, the overall learning for the two age groups decayed in a significantly different way. The differences in decay rate between the young and older subject groups suggest that there would continue to be significant age-related differences in retention at longer delay periods, since the retention in younger subjects had reached asymptote.

The decay in explicit aiming and implicit learning during the decay process was also assessed. The young subjects demonstrated a near complete retention of explicit aiming across the delay periods (Fig. $7 A$, red filled circles). This trend resembled the same results for the older subjects (Fig. $7 B$, red circles). We fitted the explicit aiming decay for both groups with the linear model and found that the linear model was not significantly different from a constant model (young group: $p=0.99, \mathrm{y}^{2}=0.0001$; older group: $p=0.52, \mathrm{p}^{2}=0.0033$ ). That is, there was near constant retention of the explicit aiming direction across the actual elapsed time. However, the retention and decay pattern for implicit learning was very different between the two groups (Fig. 7A, $B$, dark blue filled circles). For both groups, the decay of implicit learning was very similar to the decay of the overall learning (young group: time constant, $23.62 \pm 26.86 \mathrm{~s}$; older group: slope, $-0.078 \pm$ 0.020). This was expected, because, as shown above, the explicit aiming component remained fairly constant over the time delay periods. The analysis using an LMM with a random effect of subjects was performed to examine the effects of actual elapsed time, learning type, and group on the explicit and implicit learning decay. Significance was found for all three fixed effects (actual elapsed time: $F_{(1,488.16)}=49.35, p<0.0001$; learning type: $F_{(1,484.68)}=$ 156.10, $p=0.0018$; group: $\left.F_{(1,75.87)}=10.52, p=0.0018\right)$ and interaction among the three fixed effects $\left(F_{(1,484.89)}=\right.$ 9.62, $p=0.002)$. Post hoc tests showed that for both groups, implicit learning decay was significantly larger than the explicit learning decay over time (young group: $t_{\text {ratio }(484)}=3.02, p=0.0026$; older group: $t_{\text {ratio(486) }}=7.19$, $p<0.0001)$. However, there was no significant difference in the temporal angular decay of explicit aiming between the two groups $\left(t_{\text {ratio(486) }}=0.18, p>0.85\right)$ and temporal decay of the implicit learning for the older group was significantly larger than the decay of the young group $\left(t_{\text {ratio }(484)}=-4.17, p<0.0001\right)$. 

Explicit decay
Implicit decay
Overall decay
- Explicit fit
- Implicit fit
Overall fit
A

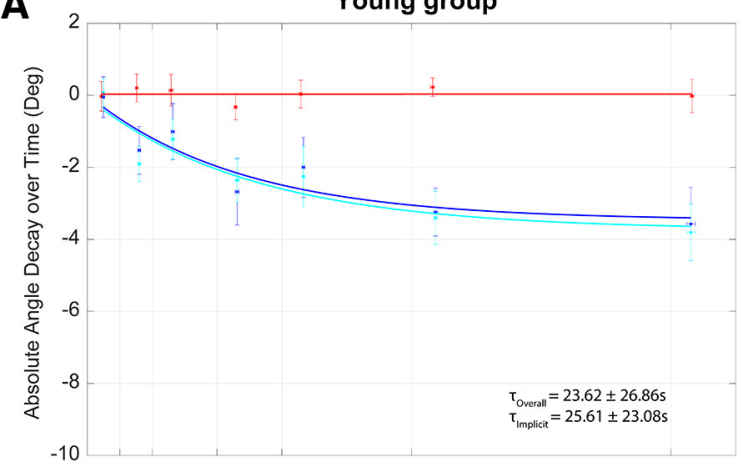

C

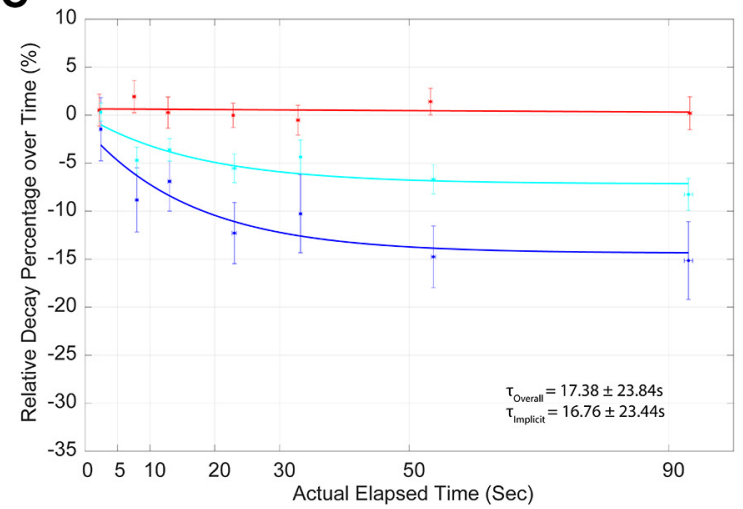

B

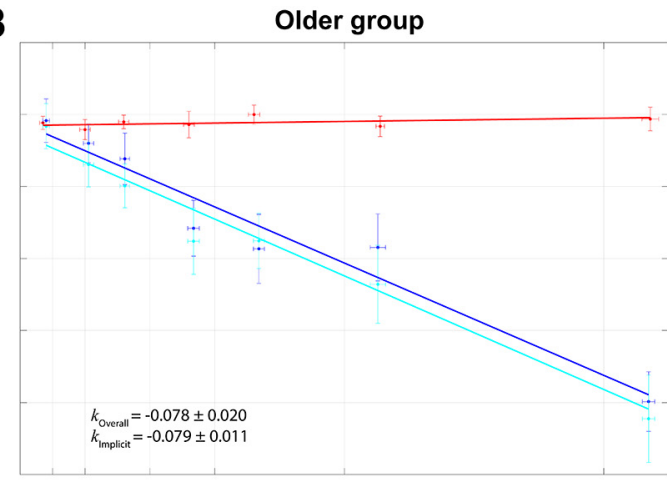

D

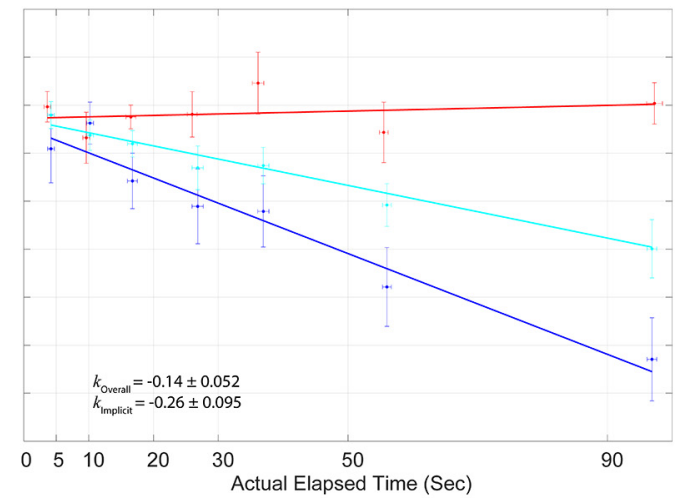

Figure 7. Temporal decay curves. $\boldsymbol{A}-\boldsymbol{D}$, The decay of implicit, explicit and overall learning as a function of the actual elapsed time is shown for young $(\boldsymbol{A}, \boldsymbol{C})$ and older $(\boldsymbol{B}, \boldsymbol{D})$ subjects. The actual elapsed time is the time interval from the end of the previous trial to the time when the selection was made for the explicit learning component, and the time interval from the end of previous trial to the movement onset for the implicit learning component. Note that the actual elapsed time was always slightly longer for the implicit learning component. The absolute retention (angular deviation) and relative retention (percentage of the adaptation at the end of preceding retraining trials) are shown on the top and bottom rows, respectively. The mean value for each respective measure (overall learning: light blue symbols; explicit aiming: red symbols; implicit learning: dark blue symbols) on the probe trial is plotted for the actual elapsed time for each respective delay period $(0,5,10,20,30,50$, and $90 \mathrm{~s})$. Each panel includes the best fit function $(\boldsymbol{A}$, $\boldsymbol{C}$, exponential for young subjects; $\boldsymbol{B}, \boldsymbol{D}$, linear for older subjects) for the decay of overall adaptation and implicit learning. Explicit learning for each group was best described by a linear function. Vertical error bars represent the SEM in the respective measures of decay, and horizontal error bars represent the SEM in the actual elapsed time.

To better understand the temporal decay for the two learning components, we also measured the relative retention compared with the end of the preceding retraining trials (Fig. $7 C, D$ ). The relative retention of the overall learning and explicit learning for both groups follows the same trends as the absolute angular retention shown in Figure 7, $A$ and $B$. (Fig. $8 A-C$ shows a direct comparison of the relative retention of explicit, implicit, and overall learning between the two groups, respectively.) Both groups had similar relative retention of the explicit learning, and the relative overall learning decay for the older group was significantly larger than that for the young group. For actual elapsed time up to $100 \mathrm{~s}$, the average decay of the overall learning for the older group $(15 \%)$ was about twice the decay for the young group (8\%). One interesting result we observed was that the relative decay of the implicit learning for both groups was about twice that of the relative decay of their overall learning. This difference is because of dividing the absolute angular decay by the implicit learning levels at the end of the retraining trials, which accounted for $\sim 50 \%$ of the overall learning (i.e., both explicit and implicit learning contributed $\sim 50 \%$ to the overall learning for both groups at the end of training; Figs. 2, 3). Thus, the relative temporal decay of the implicit learning was actually greater than the relative decay of the overall learning for both groups because of the stability of the explicit aiming (young group: time constant for the implicit decay, $16.76 \pm 23.44 \mathrm{~s}$; time constant for the overall decay, $17.38 \pm 23.84 \mathrm{~s}$; slope for the implicit decay, $-0.26 \pm 0.095$; slope for the overall decay, $-0.14 \pm$ 0.052). The same LMM with random effect of subjects was performed to examine the effects of actual elapsed time, learning type, and age group on the relative temporal decay of the explicit and implicit learning. Significance was found for the interaction among the three fixed effects $\left(F_{(1.484 .89)}=4.80, p=0.029\right)$. Post hoc tests revealed the same results that relative retention of the explicit learning was not significantly different between the two groups $\left(t_{\text {ratio(486) }}=0.36, p>0.71\right)$ and the relative temporal decay of the implicit learning for the older group was 


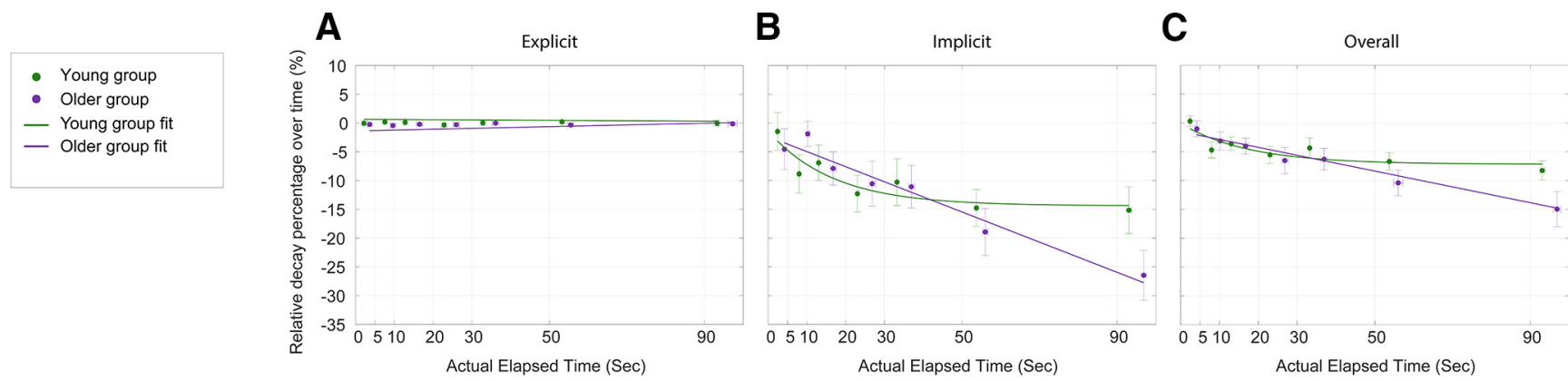

Figure 8. Comparison of the relative retention between the two age groups. $\boldsymbol{A}-\boldsymbol{C}$, The relative decay percentage for explicit $(\boldsymbol{A})$, implicit $(\boldsymbol{B})$, and overall $(\boldsymbol{C})$ learning (compared with the end of preceding retraining trials). The mean decay percentage for the two groups (young group: green symbols; older group: purple symbols) on the probe trial is plotted for the actual elapsed time for each respective delay period $(0,5,10,20,30,50$, and $90 \mathrm{~s})$, together with the best fit for each dataset (the solid lines). Vertical error bars represent SEM in the respective measures of decay, and horizontal error bars represent the SEM in the actual elapsed time.

significantly larger than the decay of the young group $\left(t_{\text {ra- }}\right.$ tio(484) $=-2.69, p=0.0074)$.

\section{Selection time during adaptation decay}

We also compared the explicit aiming selection time during the testing phase. We first examined the selection time during retraining (the average of the last three retraining trials) for each delay period for both groups. During retraining, subjects in both groups used a similar selection time compared with their selection time at the end of training, each showing no significant difference (young group: $1.63 \pm 0.14 \mathrm{~s}$; two-tailed paired $t$ test, $p=0.17, d=0.34$; older group: $3.75 \pm 0.69 \mathrm{~s}$; two-tailed paired $t$ test, $p>0.37$, $d=0.29$ ). The selection time increased for both subject groups as the delay period increased (Fig. 9A). We used an LMM with the random effect of subjects to examine the fixed effects of group and delay period on the selection time and found that the two fixed effects and their interaction were significant (effect of group: $F_{(1,37)}=16.18, p=0.00027$; effect of delay period: $F_{(6,222)}=13.69, p<0.00001$; interaction: $\left.F_{(6,222)}=3.38, p=0.0033\right)$. Post hoc tests showed that for all the delay periods $>0 \mathrm{~s}$, young subjects did not require significantly greater selection times from their selection time duration at $0 \mathrm{~s}$ (5-90 s delay periods: $p>0.05, d<0.48)$, while older subjects required a significantly longer selection time than the time they required at $0 \mathrm{~s}(5-90 \mathrm{~s}$ delay periods: $p<0.022, d>1.12)$. For all delay periods $(0-90 \mathrm{~s})$, older subjects required a significantly longer selection time than young subjects (all delay periods: $p<0.040, d>1.33$ ).

Despite the significant difference in selection time between the two groups, we were not able to distinguish between whether the older subjects need more time for choosing the explicit aiming direction or they just required more time to operate the knob compared with young subjects. Considering that both groups required significantly different selection times during retraining trials (two-tailed unpaired $t$ test, $p=0.0038, d=0.99$ ), we used the selection time in the retraining as the baseline time to operate the knob and examined whether the relative change of the selection time for each delay length remained significantly different. Thus, we took into account differences in the operation time of the knob to determine between group differences specific to forming the explicit aiming strategy. We plotted the change of the selection time for each delay period in Figure $9 A$ by subtracting the average selection time in the preceding retraining trials (Fig. 9B). The same LMM as above was performed to evaluate the difference in the change of selection time. There was a significant effect of the delay period $\left(F_{(6,222)}=12.01, p<0.00001\right)$ but no significant effect of group $\left(F_{(1,37)}=2.37, p=0.14\right)$. The interaction between the effects of group and delay period was significant $\left(F_{(6,222)}=3.20, p<0.0051\right)$. Post hoc tests showed that compared with the retraining trials, young subjects did not require significantly more time to select aiming direction (all delay periods: $p>0.18, d<0.45$ ), while older subjects needed significantly more selection time for all delay periods $>10 \mathrm{~s}$ (all cases: $p<0.0001$, $d>1.35$ ).

\section{Discussion}

\section{Influence of aging on the interaction of implicit and explicit learning mechanisms}

Although natural aging is closely associated with learning and memory impairments (Seidler et al., 2010; King et al., 2013; Cai et al., 2014), its effect on motor learning has varied. In some cases, older subjects are significantly slower to compensate for perturbations to movement. For example, Fernández-Ruiz et al. (2000) found that older subjects (age range, 50-78 years) required a longer training period to achieve the same level of prism adaptation as younger subjects (age range, 18-24 years). Similarly, in a visuomotor rotation task, Bock and Girgenrath (2006) reported older subjects (age range, 62-79 years) had a slower rate of movement recalibration compared with younger subjects (age range, 21-30 years), which corresponded with reduced performance in a cognitive reaction-time tasks. In contrast, other studies have shown little effect of aging on motor recalibration; young subjects (mean age, $23.1 \pm 4.2$ years) and older subjects (mean age, $71.1 \pm 7.8$ years) demonstrated a similar time course of adaptation in a force-field adaptation task (Trewartha et al., 2014). Similarly, Malone and Bastian (2016) showed young subjects (mean age, $22.5 \pm 2.6$ years) and older 

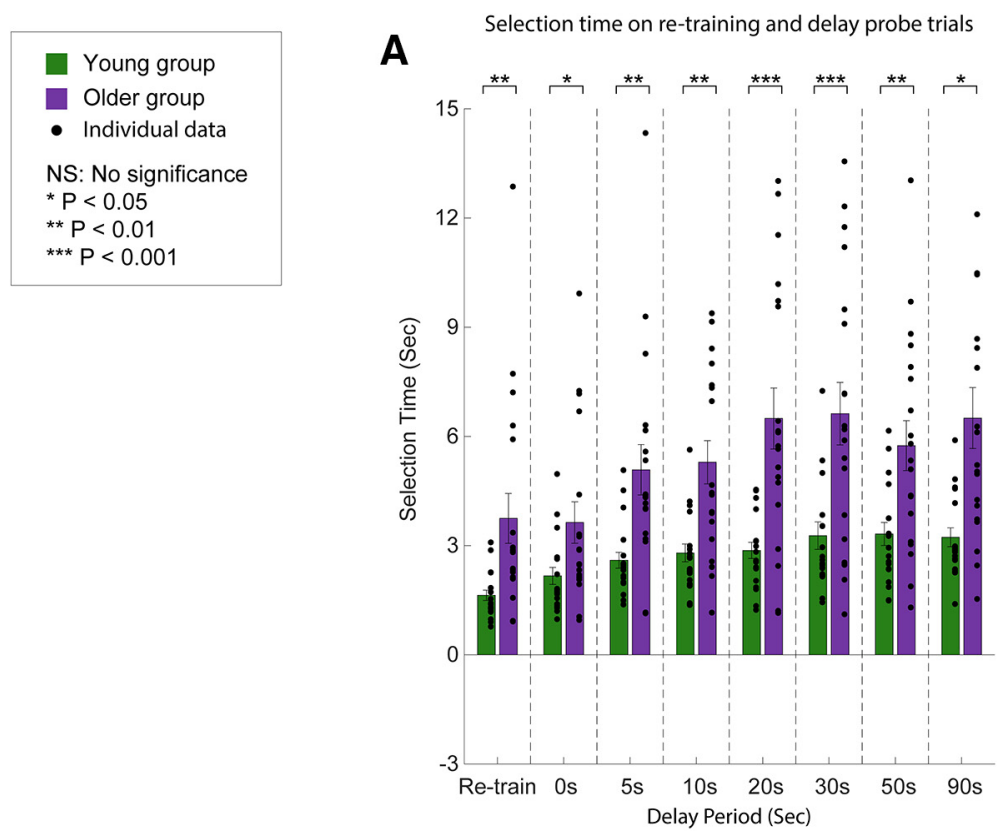

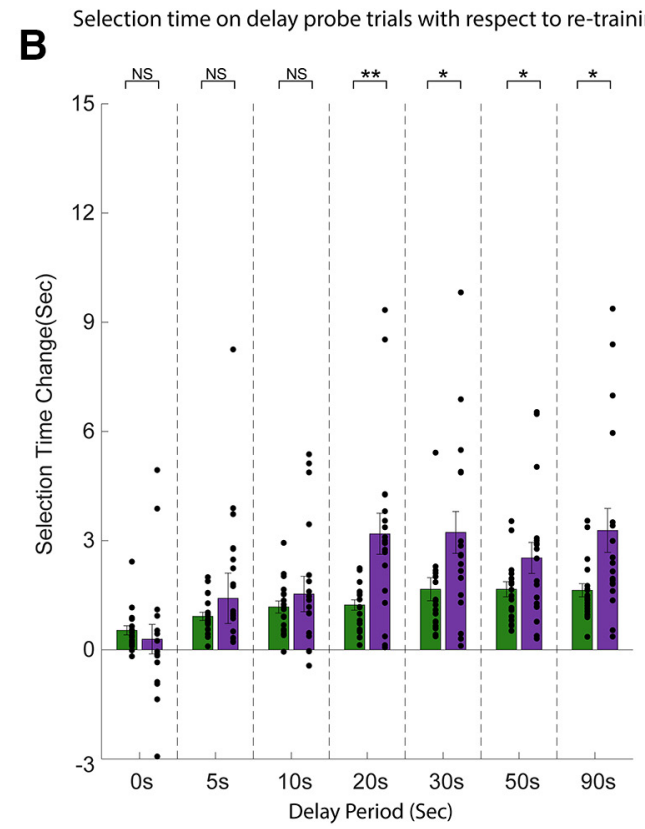

Figure 9. Selection time during the decay period. $\boldsymbol{A}$, Overall selection time for retraining trials and each delay period is shown for the young (green symbols) and older subjects (purple symbols). $\boldsymbol{B}$, The change in selection time for each delay period is shown, normalized by the selection time on the preceding retraining trials. The individual data for each subject are represented by the black filled circles next to the respective histogram. Vertical error bars represent the SEM.

subjects (mean age, $52.8 \pm 5.8$ years) adapted at similar rates in response to perturbations of gait patterns.

In the current study, we examined the respective agedependent contributions of explicit and implicit learning mechanisms to motor adaptation (Mazzoni and Krakauer, 2006; Heuer and Hegele, 2008, 2014; Lam et al., 2010; Taylor et al., 2014; Bond and Taylor, 2015; Morehead et al., 2015; De Brouwer et al., 2018; Vachon et al., 2020). Although young and older subjects adapted to the feedback rotation at somewhat similar rates (Fig. 2), older subjects consistently exhibited a higher implicit learning contribution (Fig. 3). This is largely consistent with a number of prior studies. For example, Vandevoorde and Orban de Xivry (2019) recently examined visuomotor learning in young subjects (mean age, $22.8 \pm 2.9$ years) and older subjects (mean age, $66.8 \pm 4.7$ years). Implicit learning was not impaired in older subjects, while there was a deficit in the use of cognitive strategies. Additionally, in the study by Malone and Bastian (2016) older subjects demonstrated lower adaptation curves when challenged with a distraction in the form of visual and auditory stimuli, suggesting that diverted attention hindered the ability to access and implement explicit learning strategies. Next, Buch (2003) implemented a visuomotor adaptation task in which the perturbation either increased in $11.25^{\circ}$ increments every 45 trials (gradual) or were immediately exposed to a $90^{\circ}$ perturbation (sudden). The hypothesis was that a gradual rotation would primarily entail implicit strategies, since subjects would purportedly not be consciously aware of the perturbation, while the sudden rotation was assumed to require more focus on declarative learning. Older subjects demonstrated larger errors than younger subjects during sudden adaptation, suggesting impaired explicit strategies. In contrast, both age groups exhibited similar performance in the gradual rotation task, suggesting comparable use of implicit learning mechanisms during adaptation. Finally, Wolpe et al. (2020) examined visuomotor adaptation in young subjects (age range, 18-45 years), middle-aged subjects (age range, 46-65 years), and older subjects (age range, 66-89 years), and found an agerelated decline in motor adaptation. Wolpe et al. (2020) associated this deficit with an age-related decline in explicit memory systems based on behavioral correlations with (1) explicit memory measures (e.g., the Anna Thompson Story Recall task) and (2) the reductions in gray matter volume. It is important to note that, similar to the majority of previous studies described above, Wolpe et al. (2020) did not directly assess implicit and explicit contributions to the overall adaptation, but inferred the role of explicit mechanisms by secondary tasks and correlation analyses. However, as noted above, our results are largely aligned with these conclusions; older subjects showed less of an explicit aiming contribution during learning (Fig. 2). It would be interesting to expand the findings of Wolpe et al. (2020) to determine the relationship between these identified neural areas and the differences in the shortterm temporal stability of learning shown here. For example, the authors found that age-related differences in motor adaptation were associated with gray matter volume reductions in the striatum and prefrontal cortex, but not in the cerebellum. In addition, adaptation was associated with gray matter volume in the hippocampus, a relationship that increased with age. For the 
older subjects in the current study, this would be consistent with largely intact cerebellar-based implicit learning mechanisms during training, but perhaps impaired short-term memory retention because of an age-based deficit of the hippocampus and associated connections (Albouy et al., 2008; Doyon et al., 2009; Addante, 2015).

The greater reliance on implicit learning by older subjects in the current results may be because of a disadvantage in applying explicit knowledge (Light and Singh, 1987; Durkin et al., 1995). Recently, Miyamoto et al. (2020) found that although visuomotor adaptation involves similar levels of implicit and explicit strategies, the two learning mechanisms act antagonistically, with implicit learning compensating for the variability caused by the explicit movement strategy. Our results may reflect this trade-off; although older subjects demonstrated increasing levels of explicit learning as training progressed, the level of overall adaptation remained relatively consistent. As a result, there was increased use of implicit learning when explicit mechanisms were hindered, as implicit learning may have provided a stability advantage when attempting to maintain overall adaptation. That is, the greater reliance on implicit learning mechanisms by older subjects may be beneficial in countering a possible ageassociated increase in explicit strategy-induced performance variability.

\section{Influence of aging on the temporal stability of implicit and explicit learning mechanisms}

Similar to previous studies of age-related differences in the temporal decay of performance (Loaiza and McCabe, 2013; Trewartha et al., 2014; Malone and Bastian, 2016), all subjects experienced a decrease in the overall learning with time. This is consistent with prior adaptation studies that demonstrated lower aftereffects either as a function of time or over consecutive no-feedback trials (Galea et al., 2011; Kitago et al., 2013; Taylor et al., 2014; Zhou et al., 2017). However, the pattern of temporal stability differed between the subject groups because of an age-related difference in the rate of the decay of implicit learning -exponential for young subjects and linear for older subjects. The differences in decay rate between the young and older subject groups suggests that there would continue to be significant age-related differences in retention at longer delay periods, since the retention in younger subjects had reached asymptote after $\sim 30 \mathrm{~s}$. It is important to note that these results point to an age-related deficit in the short-term temporal stability, which appears in contrast to the previous finding that older and younger subjects had similar retention of implicit and overall adaptation over a 1 min break during learning (Vandevoorde and Orban de Xivry, 2019). However, Vandevoorde and Orban de Xivry (2019) assessed this retention after a single time point, whereas we were interested in how this retention systematically changed over time. In addition, even in our study this single time point shows similar levels of decay between the two groups (Fig. 7, near the $50 \mathrm{~s}$ time point); extending the range beyond a minute (>90 s) provides a full view of the difference in the decay rates.
Furthermore, we assessed the age-based difference in stability after asymptotic levels of learning had been achieved and maintained (through retraining), not during training as in this previous study. Because of the short timescale studied (seconds to $1.5 \mathrm{~min}$ ), the current results do not contradict the observed age-related retention deficits of explicit memory functions for nonmotor tasks over longer time periods (Naveh-Benjamin et al., 2003; Kessels et al., 2007). Finally, prior studies that observed a decrease of adaptation over consecutive movement trials examined a combination of movement-dependent and time-dependent decay, rather than strictly time-dependent changes-a difference likely involving distinct neural mechanisms (Kitago et al., 2013).

There is ample evidence that short-term motor adaptation involves different learning mechanisms operating along multiple timescales (Smith et al., 2006; Körding et al., 2007; Criscimagna-Hemminger and Shadmehr, 2008; Joiner and Smith, 2008; Mawase et al., 2014; Inoue et al., 2015; McDougle et al., 2015; Alhussein et al., 2019). Sing et al. (2009) hypothesized that there is an interaction of temporally labile and temporally stable learning mechanisms (represented by fast and slow learning processes; Smith et al., 2006) that interact during adaptation. The temporally labile fast learning process decreases with the passage of time (passive decay), while the temporally stable slow component is not significantly affected with the passage of time. The slower decay of adaptation for young subjects may represent the relative stability of the temporally labile fast process, while the greater decay for older subjects may reflect the combined fading of both the slow and fast learning mechanisms. However, because adaptation to visual manipulations involves explicit cognitive strategies, we should note that state-space models that reflect fast and slow learning processes fail to capture some aspects of this type of motor recalibration (Zarahn et al., 2008). Additional studies must be conducted to develop a computational framework that fully captures these age-based behavioral results in terms of temporally labile and temporally stable learning mechanisms.

Our results presented in Figure 5 suggest that during early training the increase of the explicit aiming was related to the increase of the selection time; with longer selection time, subjects in both groups had a larger explicit aim to counter the perturbation. Although demonstrated on a significantly smaller timescale (milliseconds as opposed to seconds/minute), this benefit of increased decision time is observed in previous findings (Haith et al., 2015,2016 ) where subjects exhibited significantly greater error on trials with low preparation time compared with trials with high preparation time. Importantly, the results suggest that the time allowed to prepare the motor output differentially influences the explicit and implicit learning components-more time allows for a greater contribution of explicit strategy. The shallower relationship in early training for older subjects suggests that they required more time to form an aiming direction that young subjects were able to formulate in much less time. In addition, all subjects required more time to select an aiming direction as the delay period increased (Fig. 8). However, similar to 
training (Fig. 4), the older subjects often required twice the amount of time compared with young subjects, suggesting greater uncertainty in forming the explicit plan. This finding is consistent with prior studies (Trewartha et al., 2014; Trewartha and Flanagan, 2016), where older subjects had a longer task reaction time during an explicit memory task. Furthermore, the increased reaction time in the older group for the memory task was found to correspond with reduced explicit memory performance, demonstrating a negative relationship between reaction time and explicit memory. Trewartha and Flanagan (2016) demonstrated similar findings in the context of a weight prediction task. Our results did not show a similar temporal relationship; older subjects were able to maintain aiming accuracy although the selection time increased (Figs. $7,8)$. However, in both the training and testing phases, the longer selection time for older subjects may result from uncertainty in the aiming direction, rather than a general difficulty in making the selection. That is, there was no significant change in the explicit aiming at the end of training (Fig. 3) or over the testing phase (Fig. 7), but older subjects continued to require significantly more selection time compared with young subjects.

\section{Potential application to neurodegenerative disorders}

Our experimental paradigm may provide a basis for clinical assessments of implicit and explicit learning and memory deficits. For example, it is well known that normal aging contributes to neurochemical and physiological alterations within the brain, including cortical thinning and decreased hippocampal volume (Nyberg et al., 2012), leading to reduced working and episodic memory (Park et al., 2002; Rönnlund et al., 2005; Malouin et al., 2010; Sauzéon et al., 2016), pair association (Light et al., 2004; Old and Naveh-Benjamin, 2008), and decision-making (Cai et al., 2014). There is also evidence that proprioceptive perception and associated processing declines with age (Adamo et al., 2007; Goble et al., 2009; Piitulainen et al., 2018), which likely contribute to deficits in error-based learning and decision tasks. Treatment of neurodegenerative diseases requires a thorough understanding of how these age-related changes in sensory processing and memory stability are distinct from pathologic deficits. For example, Alzheimer's disease (AD) patients commonly experience memory deterioration and a decreased ability to make judgments and decisions (e.g., the Rey Auditory Verbal Learning Test and verbal fluency tasks; KlimkowiczMrowiec et al., 2008). In contrast, implicit learning remains largely intact, specifically in simple motor tasks (e.g., maze test, rotor pursuit, mirror tracing, and serial reaction time; Heindel et al., 1988, 1989; Knopman, 1991; Ferraro et al., 1993; Dick et al., 1995, 2001, 2003; Starkstein et al., 1997; Taylor, 1998; Rouleau et al., 2002). Our findings provide a possible standard to distinguish motor learning and retention deficits accompanying normal aging from those caused by brain disorders that progress with age. One hypothesis is that the overall short-term temporal stability of motor adaptation will be significantly impaired for AD patients because of an abnormal fading of the explicit aiming direction over time, in addition to the reduced ability to store the implicit memory component caused by hippocampal damage. Importantly, age-associated differences in the short-term temporal stability of implicit learning in the current study were observed despite similar performance on the MMSE and TMT. Thus, analyzing the temporal properties of implicit and explicit motor learning within the same behavioral context may provide a novel behavioral assay to determine the potential biomarkers of the associated structural and functional neural changes that accompany neurodegenerative disorders.

\section{Conclusions}

In this study, we examined the differences in the shortterm (seconds to $1.5 \mathrm{~min}$ ) temporal stability of explicit and implicit motor learning following adaptation of arm-reaching movements. We specifically focused on possible variations in the stability because of normal aging. In general, both young and older subjects showed similar overall learning rates of adaptation, and by the end of training there was not a significant difference in the level of explicit aiming and implicit learning between the two groups. Interestingly, over a short-term delay period (from 0 to 90 s), both young and older subjects showed almost complete retention of the explicit aiming achieved during adaptation. However, the temporal change for implicit learning was very different between subject groups. Consistent with previous work, young subjects had an exponential decrease in the implicit learning component. In stark contrast, older subjects had a linear decrease over the same time period. These results demonstrate that within the context of this motor adaptation task, normal aging reduces the shortterm stability of implicit motor learning across time, with very little impact on explicit motor learning processes.

\section{References}

Adamo DE, Martin BJ, Brown SH (2007) Age-related differences in upper limb proprioceptive acuity. Percept Mot Skills 104:12971309.

Addante RJ (2015) A critical role of the human hippocampus in an electrophysiological measure of implicit memory. Neuroimage 109:515-528.

Akaike $H$ (1974) A new look at the statistical model identification. IEEE Trans Automat Contr 19:716-723.

Albouy G, Sterpenich V, Balteau E, Vandewalle G, Desseilles M, Dang-Vu T, Darsaud A, Ruby P, Luppi PH, Degueldre C, Peigneux $P$, Luxen A, Maquet $P(2008)$ Both the hippocampus and striatum are involved in consolidation of motor sequence memory. Neuron 58:261-272.

Alhussein L, Hosseini EA, Nguyen KP, Smith MA, Joiner WM (2019) Dissociating effects of error size, training duration, and amount of adaptation on the ability to retain motor memories. J Neurophysiol 122:2027-2042.

Army Individual Test Battery (1944) Manual of directions and scoring. Washington DC: War Department, Adjutant General's Office.

Bock O (2005) Components of sensorimotor adaptation in young and elderly subjects. Exp Brain Res 160:259-263.

Bock O, Girgenrath M (2006) Relationship between sensorimotor adaptation and cognitive functions in younger and older subjects. Exp Brain Res 169:400-406.

Bond KM, Taylor JA (2015) Flexible explicit but rigid implicit learning in a visuomotor adaptation task. J Neurophysiol 113:3836-3849.

Buch ER, Young S, Contreras-Vidal JL (2003) Visuomotor adaptation in normal aging. Learn Mem 10:55-63. 
Cai L, Chan JSY, Yan JH, Peng K (2014) Brain plasticity and motor practice in cognitive aging. Front Aging Neurosci 6:31.

Clark R, Freedberg M, Hazeltine E, Voss MW (2015) Are there age-related differences in the ability to learn configural responses? Plos One 10:e0137260.

Cohen J (1988) Statistical power analysis for the behavioral sciences. New York: Routledge Academic.

Criscimagna-Hemminger SE, Shadmehr R (2008) Consolidation patterns of human motor memory. J Neurosci 28:9610-9618.

De Brouwer AJ, Albaghdadi M, Flanagan JR, Gallivan JP (2018) Using gaze behavior to parcellate the explicit and implicit contributions to visuomotor learning. J Neurophysiol 120:1602-1615.

Dennis NA, Howard JH, Howard DV (2006) Implicit sequence learning without motor sequencing in young and old adults. Exp Brain Res 175:153-164.

Dick MB, Nielson KA, Beth RE, Shankle WR, Cotman CW (1995) Acquisition and long-term retention of a fine motor skill an Alzheimer's disease. Brain Cogn 29:294-306.

Dick MB, Andel R, Bricker J, Gorospe JB, Hsieh S, Dick-Muehlke C (2001) Dependence on visual feedback during motor skill learning in Alzheimer's disease. Aging Neuropsychol Cogn 8:120-136.

Dick MB, Hsieh S, Bricker J, Dick-Muehlke C (2003) Facilitating acquisition of a continuous motor task in healthy older adults and patients with Alzheimer's disease. Neuropsychology 17:202-212.

Doyon J, Bellec P, Amsel R, Penhune V, Monchi O, Carrier J, Lehéricy S, Benali $\mathrm{H}$ (2009) Contributions of the basal ganglia and functionally related brain structures to motor learning. Behav Brain Res 199:61-75.

Durkin M, Prescott L, Furchtgott E, Cantor J, Powell DA (1995) Performance but not acquisition of skill learning is severely impaired in the elderly. Arch Gerontol Geriatr 20:167-183.

Fernández-Ruiz J, Hall C, Vergara P, Díiaz R (2000) Prism adaptation in normal aging: slower adaptation rate and larger aftereffect. Brain Res Cogn Brain Res 9:223-226.

Ferraro FR, Balota DA, Connor LT (1993) Implicit memory and the formation of new associations in nondemented Parkinson' $\mathrm{s}$ disease individuals and individuals with senile dementia of the Alzheimer type: a serial reaction time (SRT) investigation. Brain Cogn 21:163-180.

Folstein MF, Folstein SE, McHugh PR (1975) Mini-mental state: a practical method for grading the cognitive state of patients for the clinician. J Psychiatry Res 12:189-198.

Galea JM, Vazquez A, Pasricha N, Xivry J-J, Celnik P (2011) Dissociating the roles of the cerebellum and motor cortex during adaptive learning: the motor cortex retains what the cerebellum learns. Cereb Cortex 21:1761-1770.

Goble DJ, Coxon JP, Wenderoth N, Van Impe A, Swinnen SP (2009) Proprioceptive sensibility in the elderly: degeneration, functional consequences and plastic-adaptive processes. Neurosci Biobehav Rev 33:271-278.

Hadjiosif AM, Smith MA (2013) Generalization of the temporally-labile and temporally-stable components of motor memory. Soc Neurosci Abstr 39:78.23/UU17.

Haith AM, Huberdeau DM, Krakauer JW (2015) The influence of movement preparation time on the expression of visuomotor learning and savings. J Neurosci 35:5109-5117.

Haith AM, Pakpoor J, Krakauer JW (2016) Independence of movement preparation and movement initiation. J Neurosci 36:30073015.

Halekoh U, Højsgaard S (2014) A Kenward-Roger approximation and parametric bootstrap methods for tests in linear mixed modelsthe Rpackagepbkr test. J Stat Softw 59:1-32.

Heindel WC, Butters N, Salmon DP (1988) Impaired learning of a motor skill in patients with Huntington's disease. Behav Neurosci 102:141-147.

Heindel WC, Salmon DP, Shults CW, Walicke PA, Butters N (1989) Neuropsychological evidence for multiple implicit memory systems: a comparison of Alzheimer's, Huntington's, and Parkinson's disease patients. J Neurosci 9:582-587.
Heuer $\mathrm{H}$, Hegele M (2008) Adaptation to visuomotor rotations in younger and older adults. Psychol Aging 23:190-202.

Heuer H, Hegele M (2014) Age-related variations of visuo-motor adaptation beyond explicit knowledge. Front Aging Neurosci 6:152.

Inoue M, Uchimura M, Karibe A, O'Shea J, Rossetti Y, Kitazawa S (2015) Three timescales in prism adaptation. J Neurophysiol 113:328-338

Joiner WM, Smith MA (2008) Long-term retention explained by a model of short-term learning in the adaptive control of reaching. $J$ Neurophysiol 100:2948-2955.

Kennedy KM, Rodrigue KM, Bischof GN, Hebrank AC, ReuterLorenz PA, Park DC (2015) Age trajectories of functional activation under conditions of low and high processing demands: an adult lifespan fMRI study of the aging brain. Neuroimage 104:21-34.

Kessels RP, Hobbel D, Postma A (2007) Aging, context memory and binding: a comparison of "what, where and when" in young and older adults. Int J Neurosci 117:795-810.

King BR, Fogel SM, Albouy G, Doyon J (2013) Neural correlates of the age-related changes in motor sequence learning and motor adaptation in older adults. Front Hum Neurosci 7:142.

Kitago T, Ryan SL, Mazzoni P, Krakauer JW, Haith AM (2013) Unlearning versus savings in visuomotor adaptation: comparing effects of washout, passage of time, and removal of errors on motor memory. Front Hum Neurosci 7:307.

Klimkowicz-Mrowiec A, Slowik A, Krzywoszanski L, HerzogKrzywoszanska R, Szczudlik A (2008) Severity of explicit memory impairment due to Alzheimer's disease improves effectiveness of implicit learning. J Neurol 255:502-509.

Knopman D (1991) Long-term retention of implicitly acquired learning in patients with Alzheimer's disease. J Clin Exp Neuropsychol 13:880-894.

Körding KP, Beierholm U, Ma WJ, Quartz S, Tenenbaum JB, Shams $L$ (2007) Causal inference in multisensory perception. PLoS One 2: e943.

Krakauer JW, Ghilardi MF, Ghez C (1999) Independent learning of internal models for kinematic and dynamic control of reaching. Nat Neurosci 2:1026-1031.

Kuznetsova A, Brockhoff PB, Christensen RHB (2017) ImerTest package: tests in linear mixed effects models. J Stat Softw 82:1-26.

Lam WK, Maxwell JP, Masters RS (2010) Probing the allocation of attention in implicit (motor) learning. J Sports Sci 28:1543-1554.

Legendre P (2018) Model II regression. R package version 1.7-3. Vienna, Austria: R Foundation for Statistical Computing.

Lenth RV, Buerkner P, Herve M, Love J, Riebl H, Singmann H (2020) Emmeans: estimated marginal means, aka least-squares means, version-1.5.3. Vienna, Austria: $R$ Foundation for Statistical Computing

Light LL, Singh A (1987) Implicit and explicit memory in young and older adults. J Exp Psychol Learn Mem Cogn 13:541.

Light LL, Patterson MM, Chung C, Healy MR (2004) Effects of repetition and response deadline on associative recognition in young and older adults. Mem Cognit 32:1182-1193.

Loaiza VM, Mccabe DP (2013) The influence of aging on attentional refreshing and articulatory rehearsal during working memory on later episodic memory performance. Neuropsychol Dev Cogn B Aging Neuropsychol Cogn 20:471-493.

Malone LA, Bastian AJ (2016) Age-related forgetting in locomotor adaptation. Neurobiol Learn Mem 128:1-6.

Malouin F, Richards CL, Durand A (2010) Normal aging and motor imagery vividness: implications for mental practice training in rehabilitation. Arch Phys Med Rehabil 91:1122-1127.

Mawase F, Shmuelof L, Bar-Haim S, Karniel A (2014) Savings in locomotor adaptation explained by changes in learning parameters following initial adaptation. J Neurophysiol 111:1444-1454.

Mazzoni P, Krakauer JW (2006) An implicit plan overrides an explicit strategy during visuomotor adaptation. J Neurosci 26:3642-3645.

McDougle SD, Bond KM, Taylor JA (2015) Explicit and implicit processes constitute the fast and slow processes of sensorimotor learning. J Neurosci 35:9568-9579. 
Metzler-Baddeley C, Jones DK, Belaroussi B, Aggleton JP, O'Sullivan MJ (2011) Frontotemporal connections in episodic memory and aging: a diffusion MRI tractography study. J Neurosci 31:13236-13245

Miyamoto YR, Wang S, Smith MA (2020) Implicit adaptation compensates for erratic explicit strategy in human motor learning. Nat Neurosci 23:443-455.

Morehead JR, Qasim SE, Crossley MJ, Ivry R (2015) Savings upon re-aiming in visuomotor adaptation. J Neurosci 35:14386-14396.

Naveh-Benjamin M, Hussain Z, Guez J, Bar-On M (2003) Adult age differences in episodic memory: further support for an associativedeficit hypothesis. J Exp Psychol Learn Mem Cogn 29:826-837.

Nyberg L, Lövdén M, Riklund K, Lindenberger U, Bäckman L (2012) Memory aging and brain maintenance. Trends Cogn Sci 16:292305.

Old SR, Naveh-Benjamin M (2008) Differential effects of age on item and associative measures of memory: a meta-analysis. Psychol Aging 23:104-118.

Oldfield R (1971) The assessment and analysis of handedness: the Edinburgh inventory. Neuropsychologia 9:97-113.

Park DC, Lautenschlager G, Hedden T, Davidson NS, Smith AD, Smith PK (2002) Models of visuospatial and verbal memory across the adult life span. Psychol Aging 17:299-320.

Piitulainen H, Seipäjärvi S, Avela J, Parviainen T, Walker S (2018) Cortical proprioceptive processing is altered by aging. Front Aging Neurosci 10:147.

Provyn JP, Sliwinski MJ, Howard MW (2007) Effects of age on contextually mediated associations in paired associate learning. Psychol Aging 22:846-857.

Redding GM, Rossetti Y, Wallace B (2005) Applications of prism adaptation: a tutorial in theory and method. Neurosci Biobehav Rev 29:431-444.

Reitan RM, Wolfson D (1985) The halstead-Reitan neuropsychological test battery: therapy and clinical interpretation. Tucson, AZ: Neuropsychological Press.

Rönnlund M, Nyberg L, Bäckman L, Nilsson L-G (2005) Stability, growth, and decline in adult life span development of declarative memory: cross-sectional and longitudinal data from a populationbased study. Psychol Aging 20:3-18.

Rouleau I, Salmon DP, Vrbancic M (2002) Learning, retention and generalization of a mirror tracing skill in Alzheimer's disease. J Clin Exp Neuropsychol 24:239-250.

Sauzéon H, N'kaoua B, Pala PA, Taillade M, Auriacombe S, Guitton $P$ (2016) Everyday-like memory for objects in ageing and Alzheimers disease assessed in a visually complex environment: the role of executive functioning and episodic memory. J Neurophysiol 10:33-58.

Seidler RD, Bernard JA, Burutolu TB, Fling BW, Gordon MT, Gwin JT, Kwak Y, Lipps DB (2010) Motor control and aging: links to agerelated brain structural, functional, and biochemical effects. Neurosci Biobehav Rev 34:721-733.

Sing G, Najafi B, Adewuyi A, Smith MA (2009) A novel mechanism for the spacing effect: competitive inhibition between adaptive processes can explain the increase in motor skill retention associated with prolonged inter-trial spacing. In: SAC '09: proceedings of the 2009 ACM symposium on applied computing. New York: Association for Computing Machinery.

Smith MA, Ghazizadeh A, Shadmehr R (2006) Interacting adaptive processes with different timescales underlie short-term motor learning. PLoS Biol 4:e179.

Spaniol J, Madden DJ, Voss A (2006) A diffusion model analysis of adult age differences in episodic and semantic long-term memory retrieval. J Exp Psychol Learn Mem Cogn 32:101-117.

Starkstein SE, Sabe L, Cuerva AG, Kuzis G, Leiguarda R (1997) Anosognosia and procedural learning in Alzheimer's disease. Neuropsychiatry Neuropsychol Behav Neurol 10:96-101.

St-Laurent M, Abdi H, Burianová H, Grady CL (2011) Influence of aging on the neural correlates of autobiographical, episodic, and semantic memory retrieval. J Cogn Neurosci 23:4150-4163.

Synofzik M, Lindner A, Thier P (2008) The cerebellum updates predictions about the visual consequences of one's behavior. Curr Biol 18:814-818.

Talamini LM, Gorree E (2012) Aging memories: differential decay of episodic memory components. Learn Mem 19:239-246.

Taylor JA, Krakauer JW, Ivry RB (2014) Explicit and implicit contributions to learning in a sensorimotor adaptation task. J Neurosci 34:3023-3032.

Taylor R (1998) Spiral maze performance in dementia. Percept Mot Skills 87:328-330.

Trewartha KM, Flanagan JR (2016) Distinct contributions of explicit and implicit memory processes to weight prediction when lifting objects and judging their weights: an aging study. J Neurophysiol 116:1128-1136.

Trewartha KM, Garcia A, Wolpert DM, Flanagan JR (2014) Fast but fleeting: adaptive motor learning processes associated with aging and cognitive decline. J Neurosci 34:13411-13421.

Tseng Y-W, Diedrichsen J, Krakauer JW, Shadmehr R, Bastian AJ (2007) Sensory prediction errors drive cerebellum-dependent adaptation of reaching. J Neurophysiol 98:54-62.

Vachon CM, Modchalingam S, 't Hart BM, Henriques DYP (2020) The effect of age on visuomotor learning processes. PLoS One 15: e0239032.

Vandevoorde K, Orban de Xivry JJ (2019) Internal model recalibration does not deteriorate with age while motor adaptation does. Neurobiol Aging 80:138-153.

Wolpe N, Ingram JN, Tsvetanov KA, Henson RN, Wolpert DM, Rowe JB (2020) Age-related reduction in motor adaptation: brain structural correlates and the role of explicit memory. Neurobiol Aging 90:13-23.

Zarahn E, Weston GD, Liang J, Mazzoni P, Krakauer JW (2008) Explaining savings for visuomotor adaptation: linear time-invariant state-space models are not sufficient. J Neurophysiol 100:25372548.

Zhou W, Fitzgerald J, Colucci-Chang K, Murthy KG, Joiner WM (2017) The temporal stability of visuomotor adaptation generalization. J Neurophysiol 118:2435-2447. 